\title{
New York University Value-Added Galaxy Catalog: A Galaxy Catalog Based on New Public Surveys
}

\section{Citation}

Blanton, Michael R., David J. Schlegel, Michael A. Strauss, J. Brinkmann, Douglas Finkbeiner, Masataka Fukugita, James E. Gunn, et al. 2005. "New York University Value-Added Galaxy Catalog: A Galaxy Catalog Based on New Public Surveys." The Astronomical Journal 129 (6) (June): 2562-2578. doi:10.1086/429803.

\section{Published Version}

10.1086/429803

\section{Permanent link}

http://nrs.harvard.edu/urn-3:HUL.InstRepos:33461900

\section{Terms of Use}

This article was downloaded from Harvard University's DASH repository, and is made available under the terms and conditions applicable to Other Posted Material, as set forth at http:// nrs.harvard.edu/urn-3:HUL.InstRepos:dash.current.terms-of-use\#LAA

\section{Share Your Story}

The Harvard community has made this article openly available.

Please share how this access benefits you. Submit a story.

\section{Accessibility}


The Astronomical Journal, 129:2562-2578, 2005 June

(C) 2005. The American Astronomical Society. All rights reserved. Printed in U.S.A.

\title{
NEW YORK UNIVERSITY VALUE-ADDED GALAXY CATALOG: A GALAXY CATALOG BASED ON NEW PUBLIC SURVEYS ${ }^{1}$
}

\author{
Michael R. Blanton, ${ }^{1}$ David J. Schlegel, ${ }^{2}$ Michael A. Strauss, ${ }^{2}$ J. Brinkmann, ${ }^{3}$ Douglas Finkbeiner, ${ }^{2}$ \\ Masataka Fukugita, ${ }^{4}$ James E. Gunn, ${ }^{2}$ David W. Hogg, ${ }^{1}$ Želuko Ivezić, ${ }^{5}$ G. R. Knapp, ${ }^{2}$ \\ Robert H. Lupton, ${ }^{2}$ Jeffrey A. Munn, ${ }^{6}$ Donald P. Schneider, ${ }^{7}$ \\ Max Tegmark, ${ }^{8}$ and IDit Zehavi ${ }^{9}$ \\ Received 2004 October 4; accepted 2005 February 9
}

\begin{abstract}
Here we present the New York University Value-Added Galaxy Catalog (NYU-VAGC), a catalog of local galaxies (mostly below $z \approx 0.3$ ) based on a set of publicly released surveys matched to the Sloan Digital Sky Survey (SDSS) Data Release 2. The photometric catalog consists of 693,319 galaxies, QSOs, and stars; 343,568 of these have redshift determinations, mostly from the SDSS. Excluding areas masked by bright stars, the photometric sample covers $3514 \mathrm{deg}^{2}$, and the spectroscopic sample covers $2627 \mathrm{deg}^{2}$ (with about $85 \%$ completeness). Earlier, proprietary versions of this catalog have formed the basis of many SDSS investigations of the power spectrum, correlation function, and luminosity function of galaxies. Future releases will follow future public releases of the SDSS. The catalog includes matches to the Two Micron All Sky Survey Point Source Catalog and Extended Source Catalog, the IRAS Point Source Catalog Redshift Survey, the Two-Degree Field Galaxy Redshift Survey, the Third Reference Catalogue of Bright Galaxies, and the Faint Images of the Radio Sky at Twenty cm survey. We calculate and compile derived quantities from the images and spectra of the galaxies in the catalogs (for example, $K$-corrections and structural parameters for the galaxies). The SDSS catalog presented here is photometrically calibrated in a more consistent way than that distributed by the SDSS Data Release 2 Archive Servers and is thus more appropriate for largescale structure statistics, reducing systematic calibration errors across the sky from $\sim 2 \%$ to $\sim 1 \%$. We include an explicit description of the geometry of the catalog, including all imaging and targeting information as a function of sky position. Finally, we have performed eyeball quality checks on a large number of objects in the catalog in order to flag errors (such as errors in deblending). This catalog is complementary to the SDSS Archive Servers in that NYU-VAGC's calibration, geometric description, and conveniently small size are specifically designed for studying galaxy properties and large-scale structure statistics using the SDSS spectroscopic catalog.
\end{abstract}

Key words: galaxies: distances and redshifts — galaxies: photometry — galaxies: statistics

\section{MOTIVATION}

New, large galaxy data sets such as the Sloan Digital Sky Survey (SDSS; York et al. 2000) and the Two Micron All Sky Survey (2MASS; Skrutskie et al. 1997) give astronomy a view of the local universe with an unprecedented combination of completeness and detail. These surveys promise to refine our understanding of properties of galaxies, their relationships with their environment, and their evolution.

However, these catalogs consist of terabytes of data and are therefore currently too unwieldy to simply download onto a small workstation and investigate directly. Furthermore, simple matched catalogs between many surveys exist only in the form of Web interfaces such as the NASA/IPAC Extragalactic

\footnotetext{
${ }^{1}$ Center for Cosmology and Particle Physics, Department of Physics, New York University, 4 Washington Place, New York, NY 10003.

2 Princeton University Observatory, Princeton, NJ 08544.

3 Apache Point Observatory, 2001 Apache Point Road, P.O. Box 59, Sunspot, NM 88349-0059.

4 Institute for Cosmic Ray Research, University of Tokyo, Midori, Tanashi, Tokyo 188-8502, Japan.

5 Department of Astronomy, University of Washington, Box 351580, Seattle, WA 98195.

${ }^{6}$ US Naval Observatory, 3450 Massachusetts Avenue NW, Washington, DC 20392-5420.

7 Department of Astronomy and Astrophysics, Pennsylvania State University, University Park, PA 16802.

8 Department of Physics, University of Pennsylvania, Philadelphia, PA 19104.

${ }^{9}$ Department of Astronomy, University of Arizona, Tucson, AZ 85721.
}

Database (NED). ${ }^{10}$ As indispensable as such interfaces are for studying a small number of individual objects on which one desires all the knowledge in the literature, they are not ideal for huge batch jobs designed to run automatically on relatively homogeneous data sets. Finally, none of the databases interfacing to these huge catalogs yield any expression of the window function on the sky of the included surveys, leaving this critical determination to the user.

For these reasons, we have created a galaxy redshift cata$\log$ designed to aid in the study of the local universe. Early versions of this catalog have proven invaluable to the SDSS team, as they form the basis for the work of studying the luminosity function, power spectrum, correlation function, and a number of other galaxy property and galaxy clustering statistics (e.g., Blanton et al. 2003c; Tegmark et al. 2004; Zehavi et al. 2002; Hogg et al. 2003; Hoyle et al. 2005; Shen et al. 2003; Baldry et al. 2004; Pope et al. 2004). Non-SDSS investigators have also used public data extracted from this catalog to investigate galaxy properties and their evolution (e.g., Trujillo et al. 2004; Rudnick et al. 2003). The catalog is small enough (tens of gigabytes) to easily store on any modern machine, contains matches between many major public catalogs, and contains an explicit description of its window function. The current version of the catalog uses the SDSS imaging survey as its basis, matching sources in other catalogs to that master list and only tracking the

\footnotetext{
${ }^{10}$ See http://nedwww.ipac.caltech.edu.
} 
TABLE 1

Related Web Pages

\begin{tabular}{|c|c|c|c|}
\hline Survey & Description & Tools & URL \\
\hline \multirow[t]{5}{*}{ SDSS $\ldots \ldots \ldots \ldots \ldots \ldots \ldots$} & DR2 & & http://www.sdss.org/dr2 \\
\hline & Value-added catalog & vagc & http://sdss.physics.nyu.edu/vagc \\
\hline & Princeton-MIT spectroscopic reductions & idlutils & http://spectro.princeton.edu \\
\hline & Princeton-NYU photometric reductions & photoop & http://photo.astro.princeton.edu \\
\hline & $K$-corrections & kcorrect & http://sdss.physics.nyu.edu/vagc/kcorrect.html \\
\hline 2MASS ................... & Final Data Release & & http://www.ipac.caltech.edu/2mass \\
\hline $2 \mathrm{dFGRS} \ldots \ldots \ldots \ldots \ldots$ & Final Data Release & & http://msowww.anu.edu.au/2dFGRS/Public/Release \\
\hline 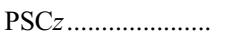 & Final Data Release & & http://www-astro.physics.ox.ac.uk/ wjs/pscz_data.html \\
\hline $\mathrm{RC} 3 \ldots \ldots \ldots \ldots \ldots \ldots \ldots$ & Ver. $3.9 \mathrm{~b}$ & & http://spider.ipac.caltech.edu/staff/hgcjr/rc3 \\
\hline ZCAT...................... & As of 2003 Nov & & http://cfa-www.harvard.edu/ huchra/zcat \\
\hline 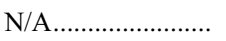 & Spherical polygon code & mangle & http://casa.colorado.edu/ ajsh/mangle \\
\hline
\end{tabular}

SDSS geometric information. In the future we plan on incorporating other surveys more fully by explicitly including their geometric information.

For most of the catalogs in the sample, we simply use the official public releases. However, we give the SDSS (of which the authors are participants) a special status in this catalog. First, it is the only survey for which we track detailed information about the window function. Second, for the SDSS we use an independent set of reductions of the public data (D. J. Schlegel et al. 2005, in preparation). These reductions are significantly improved over the reductions made available through the Data Archive Server or Catalog Archive Server on the SDSS Data Release 2 (DR2; Abazajian et al. 2004) Web site. ${ }^{11}$ The essential improvement is that errors in the large-scale relative photometric calibration of the survey have a lower variance and less structure, making the catalog more appropriate for studies of large-scale structure (as we demonstrate below). In addition, these reductions include an explicit expression for the survey geometry that the Archive Servers do not. Second, the naming and unit conventions in our catalog are different, as we describe in this paper. For these reasons, expect the quantities for objects in this catalog and the corresponding objects in the Archive Server cata$\operatorname{logs}$ to be very similar but not identical.

This paper describes only the outline and general principles of the catalog. We leave the detailed documentation to a set of regularly updated Web sites listed in Table 1 . The paper contains a short description of the geometric expressions used in the catalog and how to use them $(\S 2)$; a description of the constituent catalogs and how they are matched, focusing on the SDSS $(\S 3)$; a description of other derived quantities $(\S 4)$; the presentation of a low-redshift galaxy catalog based on these data ( $(5)$; a short description of some of the tools we have used to create this data set that might be useful to the user as well $(\S 6)$; and a summary $(\S 7)$.

Note that throughout we use the environmental variable \$VAGC_REDUX to denote the root location of the New York University Value-Added Galaxy Catalog (NYU-VAGC; see the online documentation listed in Table 1 for the actual root location). Similarly, for the large-scale structure samples we describe below, we use the environmental variable \$LSS_REDUX.

\section{SURVEY WINDOW GEOMETRIES}

In order to use galaxy surveys in a statistically meaningful way, we must describe their geometry on the sky. Doing so is important in order to determine the size of the volume surveyed, as well as to determine in what area of sky a particular type of

\footnotetext{
${ }^{11}$ See http://www.sdss.org/dr2
}

object could have been observed. However, astronomers have no standard way of expressing such geometric information. Here we describe briefly the extremely general and compact system we use, introduced by Hamilton \& Tegmark (2004). Currently, we have described the SDSS geometry using this method but have not included geometric information for the other catalogs in the NYU-VAGC.

Following Hamilton \& Tegmark (2004), we store the geometry on the sky as a set of disjoint convex spherical polygons. Spherical polygons have several advantages: they are easy to express, it is easy to determine whether a point is inside or outside, and there exist relatively simple methods to transform them into spherical harmonic components (Tegmark et al. 2002). Furthermore, they can express a variety of shapes on the sky. Since the window functions of surveys, in particular of the SDSS survey as described below, are generally complex combinations of (for example) rectangles corresponding to imaging coverage and (in the case of the SDSS) circles corresponding to the spectroscopic coverage, a flexible expression of the geometry is extremely useful. The polygons are defined as the intersection of a set of "caps" in the manner of A. Hamilton's product mangle (see Table 1). Each cap is defined as a part of a spherical surface separated out by slicing the sphere by a plane. Such a slicing cuts the sphere into two parts, and therefore, choosing a cap means picking one of these parts.

In practice, we can fully specify a cap using four numbers (three independent ones): the unit vector $\hat{\boldsymbol{x}}$ perpendicular to the plane slicing the sphere and the quantity $c_{m}= \pm(1-\cos \theta)$, where $\theta$ is the polar angle defining the angular cap radius. A positive $c_{m}$ indicates that a direction $\boldsymbol{a}$ is inside the cap if

$$
1-\boldsymbol{x} \cdot \boldsymbol{a}<\left|c_{m}\right| \cdot
$$

A negative $c_{m}$ indicates that a point is inside the cap if

$$
1-\boldsymbol{x} \cdot \boldsymbol{a}>\left|c_{m}\right| \text {. }
$$

For the value-added catalog we define the Cartesian coordinates $x_{i}$ such that

$$
\begin{gathered}
x_{0}=\cos \delta \cos \alpha, \\
x_{1}=\cos \delta \sin \alpha, \\
x_{2}=\sin \delta,
\end{gathered}
$$

where $\alpha$ and $\delta$ refer to the $\mathrm{J} 2000.0$ right ascension and declination.

The polygons are described in FITS files of the form survey_geometry.fits, where "survey" indicates which 


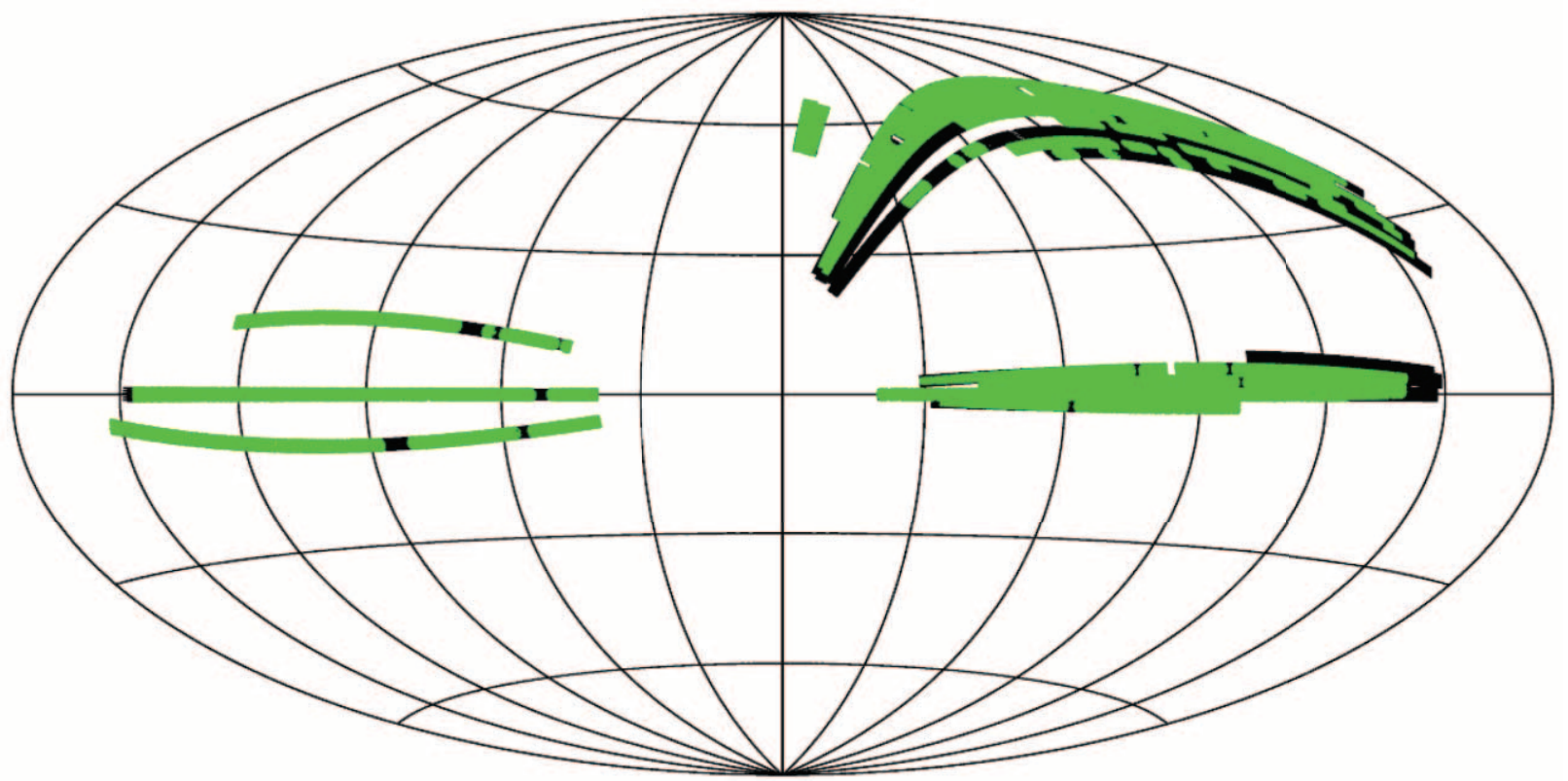

FIG. 1.-Distribution on the sky of the NYU-VAGC in equatorial coordinates. The center of this plot is the direction $\alpha=270^{\circ}, \delta=0^{\circ}$. The black areas indicate the coverage of the imaging, and the green areas indicate the location of the spectra. The survey contains $3514 \mathrm{deg}^{2}$ of imaging and $2627 \mathrm{deg}^{2}$ of spectroscopy.

survey we are describing. We also usually include ASCII versions in the standard polygon format of Hamilton \& Tegmark (2004) in an ascii subdirectory wherever the FITS version is found, ascii/survey_geometry.ply and ascii/surveygeometry_info.dat, where the "info" file contains auxiliary columns describing properties associated with each area of sky; in practice, what these properties are varies depending on the survey in question. These files contain the geometric description of each polygon. The format is described on the mangle home page (see Table 1). The description of how these structures are stored in the FITS files is on the vagc Web site (see Table 1) and tools exist in idlutils to read them into IDL structures.

It is often necessary to combine two sets of polygons describing two different surveys: for example, the SDSS imaging survey and the SDSS spectroscopic survey. In order to do so, we use a procedure known as "balkanization" (Hamilton \& Tegmark 2004). The concept is simple, although the implementation is not. Imagine plotting both sets of polygons on the same page. The lines plotted now define a new set of disjoint polygons bounded by caps, which we refer to as balkans. Each balkan is either entirely inside both surveys or inside only one survey. We can express the intersection of the two catalogs as the set of balkans entirely inside both surveys. The code presented by Hamilton \& Tegmark (2004) (see the Web site listed in Table 1) is capable of finding this set of balkans.

\section{CONSTITUENT CATALOGS}

Here we describe the set of catalogs included in the NYUVAGC: the SDSS, Faint Images of the Radio Sky at Twenty $\mathrm{cm}$ (FIRST), 2MASS, the Two-Degree Field Galaxy Redshift Survey (2dFGRS), the IRAS Point Source Catalog Redshift Survey ( $\mathrm{PSC} z$ ), and the Third Reference Catalogue of Bright Galaxies (RC3).

\subsection{SDSS}

The primary catalog is the SDSS. The SDSS is acquiring ugriz CCD imaging of $10^{4} \mathrm{deg}^{2}$ of the northern Galactic sky and, from that imaging, selecting $10^{6}$ targets for spectroscopy, most of them galaxies with $r<17.77 \mathrm{mag}$ (e.g., Gunn et al. 1998; York et al. 2000; Abazajian et al. 2003). Automated software performs all the data processing: astrometry (Pier et al. 2003); source identification, deblending, and photometry (Lupton et al. 2001); photometricity determination (Hogg et al. 2001); calibration (Fukugita et al. 1996; Smith et al. 2002); spectroscopic target selection (Eisenstein et al. 2001; Strauss et al. 2002; Richards et al. 2002); spectroscopic fiber placement (Blanton et al. 2003a); and spectroscopic data reduction. An automated pipeline measures the redshifts and classifies the reduced spectra (Stoughton et al. 2002; D. J. Schlegel et al. 2005, in preparation).

The NYU-VAGC catalog corresponds to public data up to the SDSS DR2. We include three separate catalogs from SDSS DR2: the SDSS imaging catalog, the SDSS tiling catalog (a description of the targets in the imaging catalog for which we have a welldefined completeness), and the SDSS spectroscopic catalog. Figure 1 shows the geometry of the portion of the SDSS imaging survey (DR2) released here, which includes $3514 \mathrm{deg}^{2}$ of imaging. The green points in Figure 1 show the distribution on the sky of SDSS spectra.

This list of objects includes stars, QSOs, and galaxies together. Since the typical user of this catalog will be interested in galaxies, we recommend using the VAGC_SELECT bitmask described below if they want to select Main sample-type galaxies with $m_{r}<18$. Note that the criteria on which these are selected are slightly broader than those used to target galaxies in the SDSS spectroscopy, so some of these galaxies will not have spectra for that reason. In addition, some of these "galaxies" will in fact be misidentified stars or other sources (since our criteria will necessarily be less reliable than those used by the SDSS target selection process). For maximum reliability, the user can always trim our catalog to obey the same criteria as the SDSS target selection described in Strauss et al. (2002).

\subsubsection{SDSS Imaging Catalog}

The SDSS imaging catalog presented here includes photometric reductions using photo v5_4 (R. H. Lupton et al. 2005, in preparation), and is described in Abazajian et al. (2004). 
However, unlike the data set Abazajian et al. (2004) present, it is calibrated using overlaps of SDSS runs (D. J. Schlegel et al. 2005 , in preparation). This procedure results in a more consistent large-scale calibration of the survey. In addition, as we describe below, the primary area of the survey is defined differently (and thus includes a somewhat larger fraction of the total area of the DR2 imaging).

We use some SDSS jargon below to discuss the organization of the data. As described in Stoughton et al. (2002), a "run" is a sequence number assigned to a particular drift scan observation. A "camcol" (between 1 and 6) indicates a set of ugriz CCDs in the focal plane. In each run and camcol, the imaging data are divided in the scan direction into $\sim 10^{\prime}$ "ffields" for convenience.

Only a small number of the objects in the full SDSS catalog are included here. Specifically, we include only the following:

1. A sample of objects similar to the SDSS Main galaxy sample described by Strauss et al. (2002) selected from the most recent (v5_4) of the imaging reductions. We have changed some parameters to be slightly more inclusive:

a) We extend the extinction-corrected Petrosian magnitude limit from $r=17.77$ to 18 , in order to include spectroscopic objects that would otherwise scatter outside the original flux limits as a result of changes in calibration since targeting.

b) We extend the star-magnitude separation from 0.3 to 0.2 mag (expressed as $m_{\mathrm{PSF}}-m_{\text {model }}$ in the $r$ band; see Strauss et al. 2002 for details). This change includes a small number of galaxies not included in the targeting but also introduces a number of stellar sources into the catalog.

c) We extend the bright fiber magnitude limit from $g, r$, and $i$ being more than 15,15 , and 14.5 , respectively, to $g, r$, and $i$ being more than 12 (effectively turning it off).

d) We turn off the exclusion of small, bright objects (with $R_{50}<2^{\prime \prime}$ and $\left.m_{\mathrm{P}}<15\right)$. This change results in the inclusion of a small number of binary stars.

2. The closest object within $2^{\prime \prime}$ of the position of each SDSS spectrum. In this match, we in fact give priority to objects that pass the previous criterion to be a spectroscopic galaxy target in the latest reductions. One might worry that this would introduce some sort of flux bias in the sample, since these targets are bright relative to the typical object, but in fact any pairs of objects with separations less than $2^{\prime \prime}$ are likely to be spurious in any case. Thus, taking the brightest one is usually the correct choice.

3 . The closest object within $2^{\prime \prime}$ of the position of each Main sample, QSO, or Luminous Red Galaxy spectroscopic target from the target version of the reductions that was fed to the tiling software (a "tiled target"), whether or not they had spectra taken. These targets were selected on the basis of earlier reductions of the imaging that differ in significant ways from the latest reductions.

We have retained the Petrosian half-light $r$-band surface brightness limit at $\mu_{50}=24.5$. Note that the new objects included by the extensions described above are unlikely to have spectra.

In fact, because of catalog errors, there remain a handful of pairs of objects within $2^{\prime \prime}$ of each other (12 out of 693,331). After removing these duplicates, we include one observation of each object in the file \$VAGC_REDUX/object_sdss_imaging.fits, which contains a trimmed set of the photometric measurements (the exact list is described on the Web site listed in Table 1). We use the environmental variable \$VAGC_REDUX to denote the root location of the NYU-VAGC; see the online documentation for the actual root location. In addition, we store all the photometric information for this set of objects in another set of files of the form \$VAGC_REDUX/sdss/parameters/calibObj-[run] [camcol].fits, where, as indicated, objects in separate "runs" and "camcols" are kept in separate files. The object_sdss_ imaging file contains the run and camcol for each object, as well as its zero-indexed position in the corresponding calibObj file, allowing quick access to the full photometric information.

The geometry of the SDSS imaging survey is in the file \$VAGC_REDUX/sdss/sdss_imaging_geometry.fits, stored in the form of spherical polygons. We create a list of polygons that describe the primary area of each imaging field. For each polygon, we give the SDSS imaging field (that is, its run, camcol, and field) that we consider "primary" for the purpose of resolving duplicate observations.

We should note here some points about the quantities in the SDSS data we present (some of which differ from data distributed by the Archive Servers):

1. All fluxes are given in "nanomaggies" $f$, which represent the flux (multiplied by $10^{9}$, as the prefix "nano" implies) relative to that of the $\mathrm{AB}$ standard source with $f_{\nu}=3631 \mathrm{Jy}$ (Oke $\&$ Sandage 1968). They are related to standard astronomical magnitudes $m$ by the formula

$$
m=22.5-2.5 \log _{10} f
$$

2. The zero points of the system are the same as for the data on the Archive Servers, so that the conversion to AB magnitudes is the same as that given by Abazajian et al. (2004).

3 . Uncertainties are expressed in terms of the inverse variance (usually used for calibrated quantities, with the suffix _ IVAR) or in terms of the standard deviation (usually used with uncalibrated quantities, with the suffix ERR).

4. The column VAGC_SELECT is a bitmask that yields information on how each imaging object was selected, with the following bits:

0: Near tiled target.

1: Near spectrum.

2: Passed the Main sample galaxy criteria (with the adjustments listed above).

Thus, an object that passed the galaxy criteria, was near a tiled target, and was near a spectrum would have bits 0,1 , and 2 all set, resulting in a numerical value of 7 for VAGC_SELECT.

5. In addition to the local sky determination (the $100^{\prime \prime} \times 100^{\prime \prime}$ median smoothed sky in skyux), we provide the sky estimate for the current 9.' $8 \times 13.5$ field as a whole ("global" sky) in the parameter psp_skyflux (in nanomaggies $\operatorname{arcsec}^{-2}$ ).

6. A crude bulge-to-disk decomposition exists for each object processed by photo, which simply consists of taking the best-fit de Vaucouleurs model and the best-fit exponential model, linearly combining them, and refitting for the amplitudes of the models (see Abazajian et al. 2004 for details). The fraction of the flux assigned to the de Vaucouleurs model (the "bulge fraction," if you will allow it) is put in the column called fracpsf.

In addition to these changes of form, there is a fundamental difference between the Princeton reductions used here and the data available on the SDSS Archive Servers: the relative photometric calibration (D. J. Schlegel et al. 2005, in preparation). The Archive Server reductions use the calibration procedure described in Abazajian et al. (2003), which involves comparing counts measured on the $2.5 \mathrm{~m}$ telescope to those measured on a nearby $0.5 \mathrm{~m}$ photometric telescope on a slightly different filter system and to the fluxes of a set of primary standard stars (on yet another filter system). Instead of this procedure, D. J. Schlegel 
et al. (2005, in preparation) take advantage of the wide angular baseline provided by the drift scanning and of the large number of overlapping observations. This combination results in many exposures of the same stars taken on different nights. One can then use these multiple observations to fit for the calibration parameters (system response, air mass term, flat fields) as a function of time by minimizing the differences between the resulting inferred fluxes of the multiply observed stars. This procedure, denoted "ubercalibration," takes advantage of the fact that the system is photometrically stable within each drift scan run and uses that to tie all the runs together using their overlapping observations.

As a demonstration that the procedure results in a lower variance in large-scale errors in the calibration, consider Figure 2. The gray scale in the top two panels shows the $r-i$ color of the bluest stars in the magnitude range $16<m_{r}<18.5$ in each contiguous set of 20 fields in each run of the SDSS (all magnitudes extinction-corrected according to the dust maps of Schlegel et al. [1998]). We only show one section of the SDSS coverage on the northern equator, but similar results hold elsewhere. This blue-tip color varies smoothly across the sky as a result of metallicity gradients in the Galactic stellar halo but has little small-scale structure. Figure 2 (top) shows this quantity for data we have calibrated to the SDSS standard system using the photometric telescope (although these results are not identical to those in the SDSS Archive). Figure 2 (middle) shows the same for the ubercalibrated data. Both panels reveal the dependence of stellar metallicity on Galactic latitude, as well as some large-scale features in the stellar distribution. Clearly, the stripy variations in Figure 2 (top), which are errors in the SDSS calibration, are greatly reduced in Figure 2 (middle) (although not eliminated). The $5 \sigma$-clipped standard deviation in $r-i$ color over the whole SDSS area is reduced from 0.02 to $0.01 \mathrm{mag}$ between the photometric telescope calibration and the ubercalibration (these numbers include the variation of the stellar populations). The rms variations of $r-i$ within several degree scale patches is about $0.007 \mathrm{mag}$. These results suggest that ubercalibration is a significant improvement over the standard calibration and that the calibration is good to about $1 \%$. The SDSS is taking long scans across the entire survey in the northern Galactic cap, as well as scans that connect the three separated stripes in the southern Galactic cap, that will reduce the systematic errors even further.

Our Web site (see Table 1) has full documentation of the structure of the files described in this and subsequent sections and of all the parameters they contain.

\subsubsection{SDSS Tiling Catalog}

The primary SDSS spectroscopic program proceeds in the following manner: On the basis of a set of images for which we have selected targets (using the algorithms in Strauss et al. [2002], Eisenstein et al. [2001], Richards et al. [2002], and Stoughton et al. [2002]), the SDSS defines a "tiling region." For example, for tiling region 7 in the SDSS, Figure 3 shows the region we defined. Given the tiling region and the set of targets within it, we determine the location of spectroscopic tiles of radius 1.49 and decide to which targets to assign fibers (Blanton et al. 2003a). This procedure defines a set of 1.49 radius circles on the sky corresponding to the tiles. The intersection of the rectangles describing the tiling region and the circles describing the tiles defines the geometry of the tiling region. In this geometry we define "sectors," each of which consists of a set of spherical polygons that could have been observed by a unique set of tiles. These sectors are the appropriate regions on which to define the completeness of the survey. See the DR2 Web site $^{12}$ for more complete documentation on tiling. In Figure 3 we have given each sector a different shade of gray.

The union of the tiling regions defines the geometry of the spectroscopic survey as a whole. Note that this geometry is not, generally, as simple as the total area covered by the tiles. For example, some regions are within 1.49 of the center of a tile, but the tile was created before spectroscopic targets for that region had been selected. This fact of life results in gaps in the survey that we track in our geometric description of the survey.

The set of polygons describing the tiling geometry is in \$VAGC_REDUX/sdss/sdss_tiling_geometry.fits. This file yields the sector to which each polygon belongs. The properties of the sectors are given in \$VAGC_REDUX/sdss/sdss_sector List.par, which yields which tile each sector belongs to. Finally, the centers of each tile are given in \$VAGC_REDUX/sdss/ tileFull.par.

We match the set of objects that we input into the tiling program to the nearest imaging object within $2^{\prime \prime}$ in the object_ sdss_imaging catalog and put the results in the file \$VAGC_ REDUX/object_sdss_tiling.fits. Each entry in this file refers to the corresponding entry in the object_sdss_imaging file; e.g., row number 3 in one file refers to the same object as row number 3 in the other. The full set of tiled objects (including those that do not match any of the imaging objects) is included in the file \$VAGC_REDUX/sdss/sdss_tiling_catalog.fits. The object_sdss_tiling.fits file has the column sdss_ tiling-tag-primary, which gives the zero-indexed row number of the object in the sdss_tiling_catalog.fits file. Unmatched objects have sdss_tiling_tag_primary $=-1$ (and the rest of the columns for such rows are set to appropriate null values).

The reader may wonder why there would be any unmatched objects. The reason is that the photometric reduction code has changed over time; in particular, the deblending algorithm has changed. For this reason, there are occasionally objects found in an old reduction that have no corresponding object within $2^{\prime \prime}$ in the new reductions, because the set of detected pixels in that region has been deblended differently by the two versions of the code. Again, the structure and contents of these files are described on the Web site.

\subsubsection{SDSS Spectroscopic Catalog}

For this catalog we use the reductions of the SDSS spectroscopic data performed by D. J. Schlegel et al. (2005, in preparation) using their reduction code idlspec $2 \mathrm{~d}$, which extracts the spectra and finds the redshifts. The redshifts found by idlspec2d are almost always (over $99 \%$ of the time for Main galaxy sample targets) identical to the redshifts found by an alternative pipeline used for the SDSS Archive Servers (M. SubbaRao et al. 2005, in preparation).

We match the set of objects for which we have SDSS spectra to the nearest imaging object within $2^{\prime \prime}$ in the object_sdss_ imaging catalog and put the results in the file \$VAGC_REDUX/ object_sdss_spectro.fits. Each entry in this file refers to the corresponding entry in the object_sdss_imaging file; e.g., row number 3 in one file refers to the same object as row number 3 in the other. The full set of spectra (including those that do not match any of the imaging objects) is included in the file \$VAGC_REDUX/sdss/sdss_spectro_catalog. fits. The object_sdss_spectro.fits file has the column sdss_ spectro_tag-primary, which gives the zero-indexed row

\footnotetext{
12 See http://www.sdss.org/dr2/products/tiling.
} 

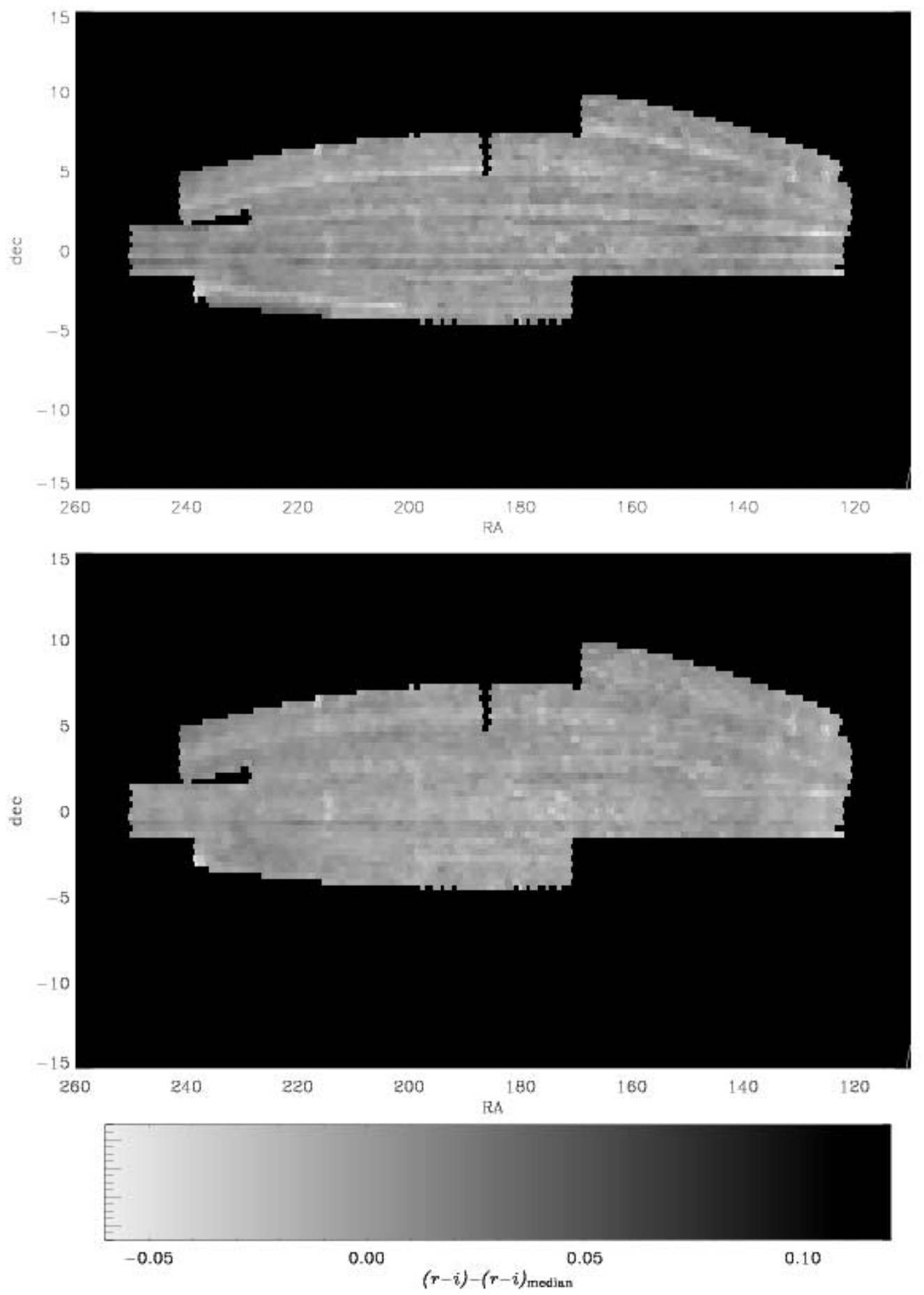

FIG. 2.-Top, middle: The $r-i$ color of the bluest stars in the magnitude range $16<m_{r}<18.5$ in each contiguous set of 20 fields in each run of the SDSS (all magnitudes are extinction-corrected according to the dust maps of Schlegel et al. [1998]). Bottom: Calibration of the scale. Only the northern equatorial data are shown. The top panel shows this quantity for the data calibrated to the SDSS standard system using the photometric telescope. The middle panel shows the same for the ubercalibrated data, as described in the text. 


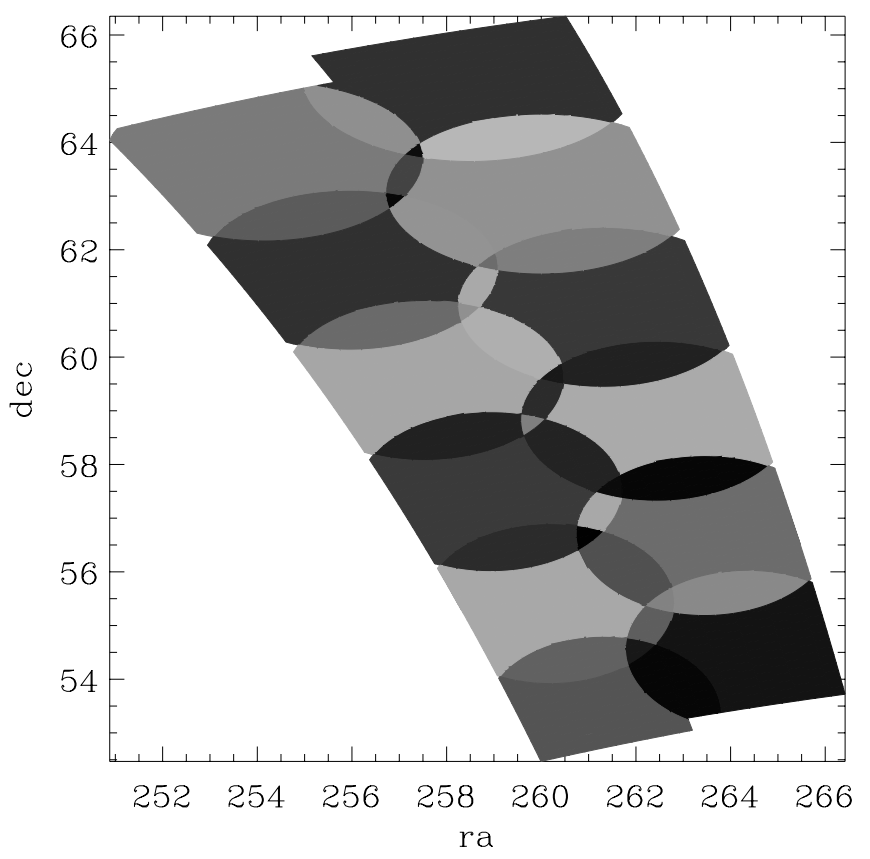

FIG. 3.-Example of a "tiling region" in the survey (tiling region 7). Each shade of gray indicates the coverage of a given "sector" (as defined in the text, a region covered by a unique set of tiles).

number of the object in the sdss_spectro_catalog. fits file. Unmatched objects have sdss_spectro_tag_primary $=-1$ (and the rest of the columns for such rows are set to appropriate null values). Note that in addition to the issues regarding deblending in different reductions mentioned in $\S 3.1 .2$, a number of the spectra are sky spectra that are placed randomly on the sky and will never correspond across reductions. We do not provide any geometric description of the catalog beyond the locations of each fiber (each one has a $3^{\prime \prime}$ diameter). The quantities in these files are documented at the spectroscopic reduction Web site (see Table 1).

\subsection{FIRST}

Using the Very Large Array, the FIRST survey (Becker et al. 1995) has mapped 10,000 deg ${ }^{2}$ of the northern sky, overlapping the SDSS with a detection limit of about $1 \mathrm{mJy}$ and a resolution of $5^{\prime \prime}$. For each object in object_sdss_imaging, we find the matching object in the FIRST catalogs within $3^{\prime \prime}$. The columns with the prefix FIRST in the files \$VAGC_REDUX/sdss/ parameters/calibObj-\$run-\$camcol.fits contain the FIRST results. The columns are described in detail in the Princeton photometric reduction Web site listed in Table 1.

\section{3. $2 M A S S$}

2MASS (Cutri et al. 2000) is an all-sky map in $J, H$, and $K_{s}$. Two catalogs have been developed from this map; the Point Source Catalog (PSC; complete to roughly $K_{S} \sim 15$, Vega relative) and the Extended Source Catalog (XSC), that is, the galaxy catalog (complete to roughly $K_{s} \sim 13.5$ ). For each object in object_sdss_imaging, we find the matching object within $3^{\prime \prime}$. The columns with the prefix TMASS in the files \$VAGC_ REDUX/sdss/parameters/calibObj-\$run-\$camcol.fits contain the 2MASS PSC data. The columns are described in detail in the Princeton photometric reduction Web site listed in Table 1.

In addition, we match the 2MASS XSC (described in the explanatory supplement to the 2MASS All Sky Data Release; see Table 1; Cutri et al. 2000) to objects within $3^{\prime \prime}$ in object_

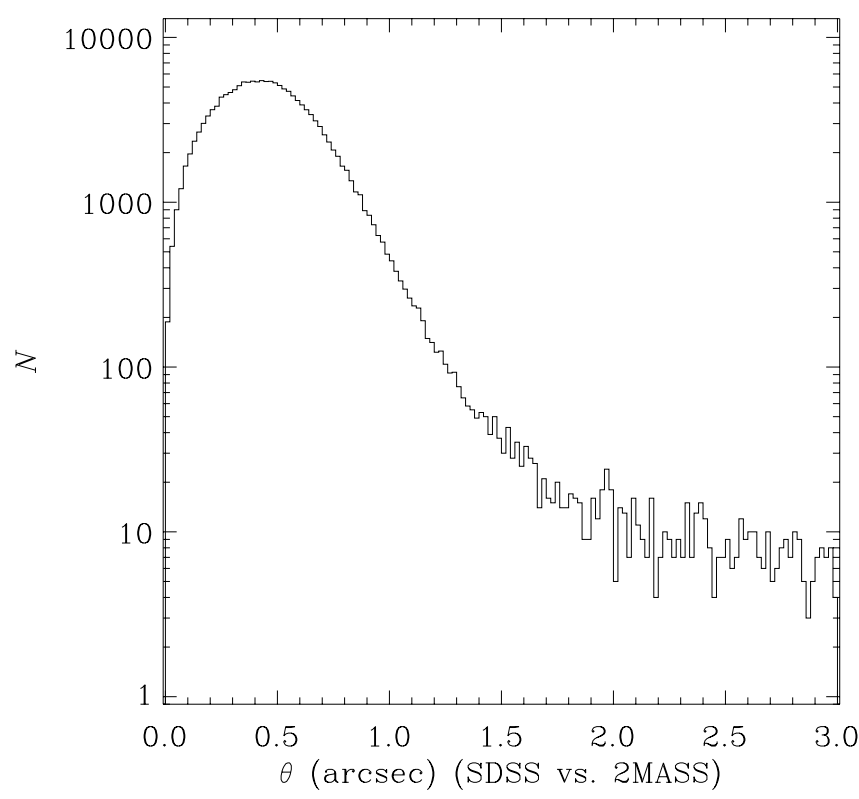

FIG. 4.-Distribution of angular distances between the matched objects in the SDSS and 2MASS (in the object_sdss_imaging and object_twomass catalogs). The astrometry of the surveys agrees well.

sdss_imaging and put the results in the file \$VAGC_REDUX/ object_twomass.fits. Each entry in this file refers to the corresponding entry in the object_sdss_imaging file; e.g., row number 3 in one file refers to the same object as row number 3 in the other. The full set of 2MASS XSC objects (including those that do not match any of the imaging objects) is included in the files \$VAGC_REDUX/twomass/twomass_catalog_000.fits, \$VAGC_REDUX/twomass/twomass_catalog_001.fits, \$VAGC_ REDUX/twomass/twomass_catalog_002.fits, and \$VAGC_REDUX/ twomass/twomass_catalog_003.fits. These files contain a subset of the columns listed by Cutri et al. (2000). Most notably, below we use the "extrapolated" galaxy magnitudes from these files, as described by Jarrett et al. (2003).

We have not converted the numbers in these files from their original Vega relative meaning. Where necessary below, we use the conversions to $\mathrm{AB}$,

$$
\begin{aligned}
J_{\mathrm{AB}} & =J_{\mathrm{Vega}}+0.91, \\
H_{\mathrm{AB}} & =H_{\mathrm{Vega}}+1.39, \\
K_{s, \mathrm{AB}} & =K_{s, \text { Vega }}+1.85,
\end{aligned}
$$

calculated by the kcorrect v3_2 code, presented by Blanton et al. (2003b), using the filter curves of Cutri et al. (2000) and the theoretical Vega flux presented by Kurucz (1991). Figure 4 shows the distribution of match distances between the SDSS and 2MASS catalogs, showing the agreement in the astrometry between them (Pier et al. 2003; Finlator et al. 2000).

\section{4. $2 d F G R S$}

The 2dFGRS (Colless et al. 2001) is a galaxy redshift survey using the $2 \mathrm{dF}$ multiobject spectrograph, targeted off of the Automated Plate Measuring survey (Maddox et al. 1990). We match the 2dFGRS Final Data Release to objects within $4^{\prime \prime}$ in object_sdss_imaging and put the results in the file $\$$ VAGC_ REDUX/object_twodf.fits. Each entry in this file refers to the corresponding entry in the object_sdss_imaging file; e.g., row number 3 in one file refers to the same object as row number 3 in the other. The full set of 2 dFGRS objects (including those 


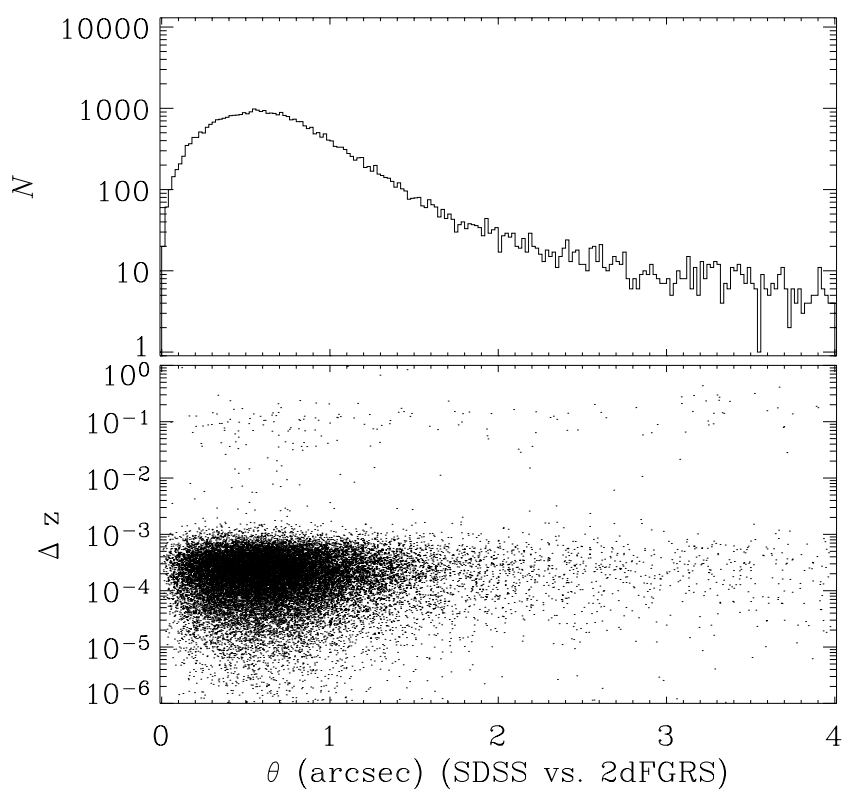

FIG. 5.-Top: Distribution of angular distances between the matched objects in the SDSS and 2dFGRS (in the object_sdss_imaging and object_twodf catalogs). Bottom: Absolute redshift differences vs. angular distances for the same matches. A small number (170) of these galaxies are large outliers. In all such cases the SDSS pipeline either yields a clearly correct redshift based on an eyeball examination of the spectra or flags the spectrum as problematic with the ZWARNING flag.

that do not match any of the imaging objects) is included in the files \$VAGC_REDUX/twodf/twodf_catalog.fits.

Figure 5 (top) shows the distribution of angular separations of our matches, and Figure 5 (bottom) (for objects with redshifts in both catalogs) the (absolute) difference between the SDSS and $2 \mathrm{dFGRS}$ redshifts versus the angular distance. We limit our comparison to 2dFGRS redshifts with QUALITY $\geq 3$ (the recommended criterion for a reliable redshift). There are around 27,000 objects with redshifts in both catalogs; 94 of these are large redshift discrepancies $(|\delta z|>0.01)$. For about 83 of these discrepancies, the SDSS redshift is clearly correct on the basis of an eyeball inspection of the extracted spectrum. For the remaining 11, the SDSS redshift is flagged as poor by the spectroscopic reduction software (using the ZWARNING flag described on the spectroscopic reduction Web site listed in Table 1).

\subsection{IRAS PSCz}

The PSC $z$ is a redshift catalog of point sources in the IRAS survey (Saunders et al. 2000). Given the resolution of the IRAS survey, we have matched each source to the nearest object within $40^{\prime \prime}$ in the object_sdss_imaging file, putting the results in the file \$VAGC_REDUX/object_pscz.fits. Each entry in this file refers to the corresponding entry in the object_sdss_imaging file; e.g., row number 3 in one file refers to the same object as row number 3 in the other. The full set of $\mathrm{PSC} z$ objects (including those that do not match any of the imaging objects) is included in the files \$VAGC_REDUX/pscz/pscz_catalog.fits.

Figure 6 (top) shows the distribution of angular separations of our matches, and Figure 6 (bottom) (for objects with redshifts in both catalogs) the (absolute) redshift difference versus the angular distance. The redshift matches are good in all cases.

\section{6. $R C 3$}

RC3 is a catalog of nearby galaxies developed by de Vaucouleurs et al. (1991). Because of the size of these galaxies

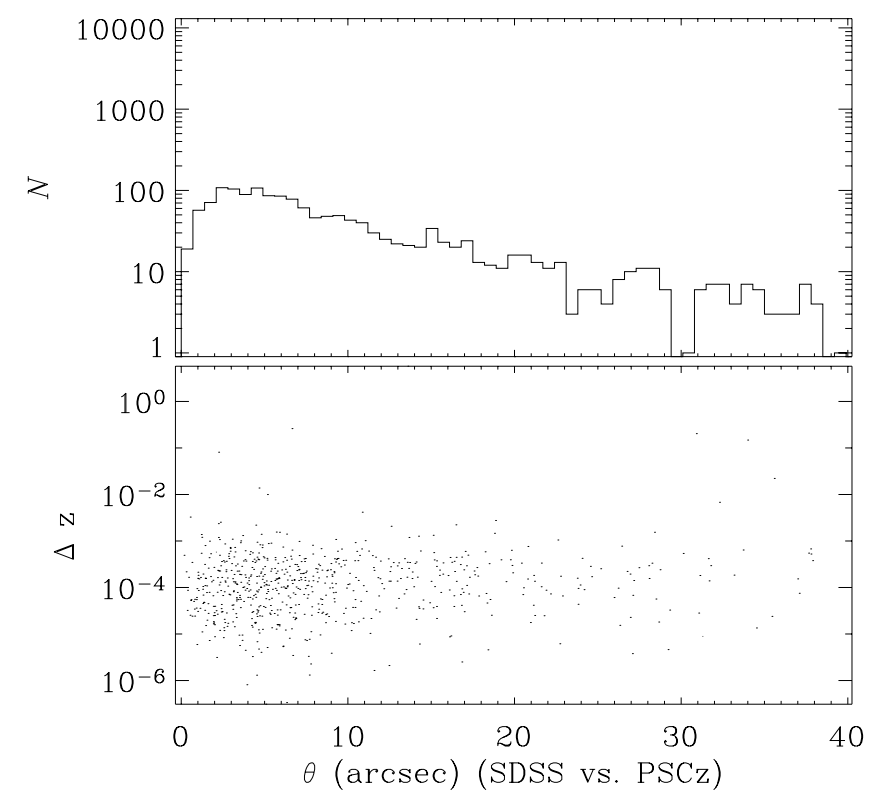

FIG. 6.-Similar to Fig. 5 but for the SDSS and PSC $z$ (the object_sdss_ imaging and object_pscz catalogs). The redshift differences are small even for objects with large differences in astrometry, indicating that the matches are reliable out to $40^{\prime \prime}$.

(and the fact that locations in the RC3 are occasionally listed only to the nearest arcminute), we have matched each source to the nearest object within $45^{\prime \prime}$ in the object_sdss_imaging file, putting the results in the file \$VAGC_REDUX/object_ rc3. fits. Each entry in this file refers to the corresponding entry in the object_sdss_imaging file; e.g., row number 3 in one file refers to the same object as row number 3 in the other. The full set of RC3 objects (including those that do not match any of the imaging objects) is included in the files \$VAGC_REDUX/rc3/ rc3_catalog.fits.

Figure 7 (top) shows the distribution of angular separations of our matches, and Figure 7 (bottom) (for objects with redshifts

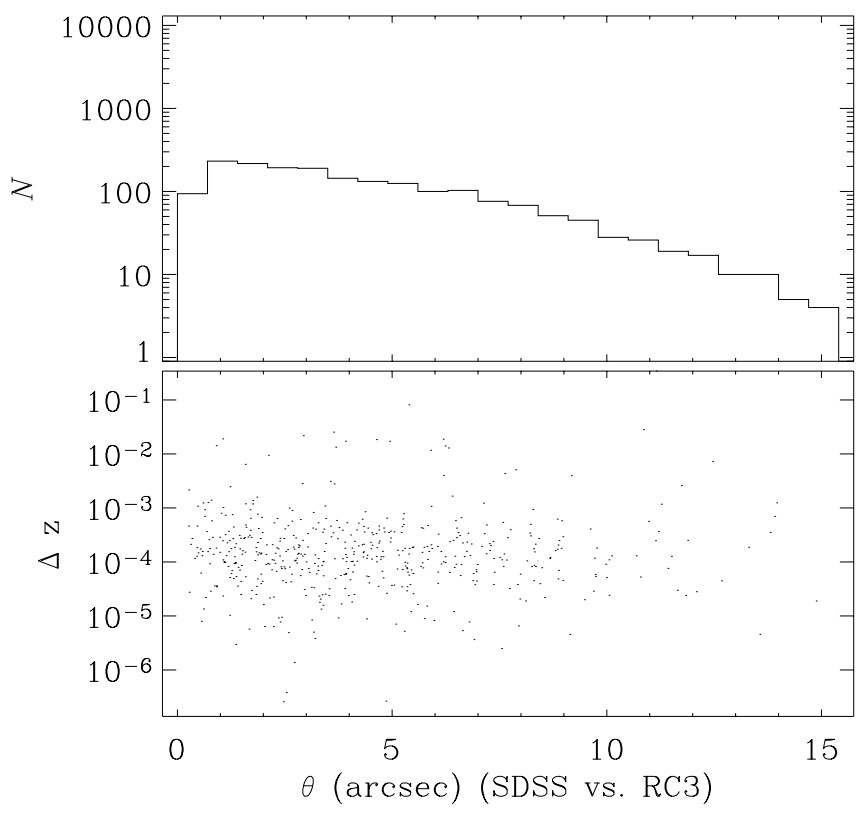

Fig. 7.- Similar to Fig. 5 but for the SDSS and RC3 (the object_sdss_ imaging and object_rc3 catalogs). The matches appear reliable out to $15^{\prime \prime}$. 
in both catalogs) the (absolute) redshift difference versus the angular distance.

\section{ADDITIONAL QUANTITIES}

In addition to matches to external catalogs, we provide some extra quantities measured from the NYU-VAGC catalog.

\subsection{Collision "Corrections"}

For the purposes of large-scale structure statistics with the SDSS, it is necessary to account for the fact that some galaxies are missing in the spectroscopic sample as a result of collided fiber constraints (no two fibers on the same tile can be placed more closely than $55^{\prime \prime}$ ). We can do so using the following procedure:

1. Group the galaxies according to a friends-of-friends procedure with a $55^{\prime \prime}$ linking length.

2. For each galaxy that does not have a redshift in the SDSS data, ask whether there is a galaxy (or galaxies) in its group with a redshift.

3. If so, assign to the galaxy without a redshift that of the galaxy in the group that is closest on the sky and that has a redshift.

We put the results of this procedure into a file \$VAGC_REDUX/ collisions/collisions nearest. fits. About $5 \%-6 \%$ of galaxies brighter than the flux limit need to be and can be assigned a redshift using this criterion, as found previously by Zehavi et al. (2002). Judging from the cases that could have been corrected but in fact had a redshift, about $60 \%$ of the corrected cases are within $10 h^{-1} \mathrm{Mpc}$ of the correct redshift. Figure 8 shows the distribution of redshift separations and angular separations of such galaxies in the top panel and the histogram of redshift separations in the bottom panel.

We have also implemented a slight variant of this procedure, in which we also require that the photometric redshift of the galaxy (determined using kcorrect v3_2; Blanton et al. 2003b) on the collided fiber be within 0.05 of the spectroscopic redshift that we want to assign to it. There is very little difference in the results; we include them in the file \$VAGC_REDUX/collisions/ collisions.photoz.fits. About $9 \%$ of the objects are corrected, about $71 \%$ of which are likely to be within $10 \mathrm{~h}^{-1}$ of the correct redshift (on the basis of the cases that could have been corrected but in fact had a redshift).

Finally, for completeness we include the corresponding files without any corrections at all: \$VAGC_REDUX/collisions / collisions.none.fits.

\subsection{K-Corrections}

We use the $K$-correction software kcorrect v3_2 (Blanton et al. 2003b) to determine $K$-corrections for all the objects in the NYU-VAGC. We treat them all as if they are normal galaxies; thus, the $K$-corrections are not going to be appropriate for QSOs.

In the directory \$VAGC_REDUX/kcorrect we provide these estimates of the $u g r i z J H K_{s} K$-corrections and absolute magnitudes of each object (using a $\Omega_{0}=0.3, \Omega_{\Lambda}=0.7$ cosmology with $H_{0}=100 h \mathrm{~km} \mathrm{~s}^{-1} \mathrm{Mpc}^{-1}$ for $\left.h=1\right)$. The $J H K_{s}$ fluxes all come from the 2MASS XSC extrapolated magnitudes (Jarrett et al. 2003) and have been converted from the Vega system to the $\mathrm{AB}$ system as described in $\S 3.3$. The files also contain the Galactic extinction-corrected $\mathrm{AB}$ nanomaggies for each object. We provide these for each set of collision corrections, for different types of SDSS flux measurements, and for different rest-frame bandpasses.

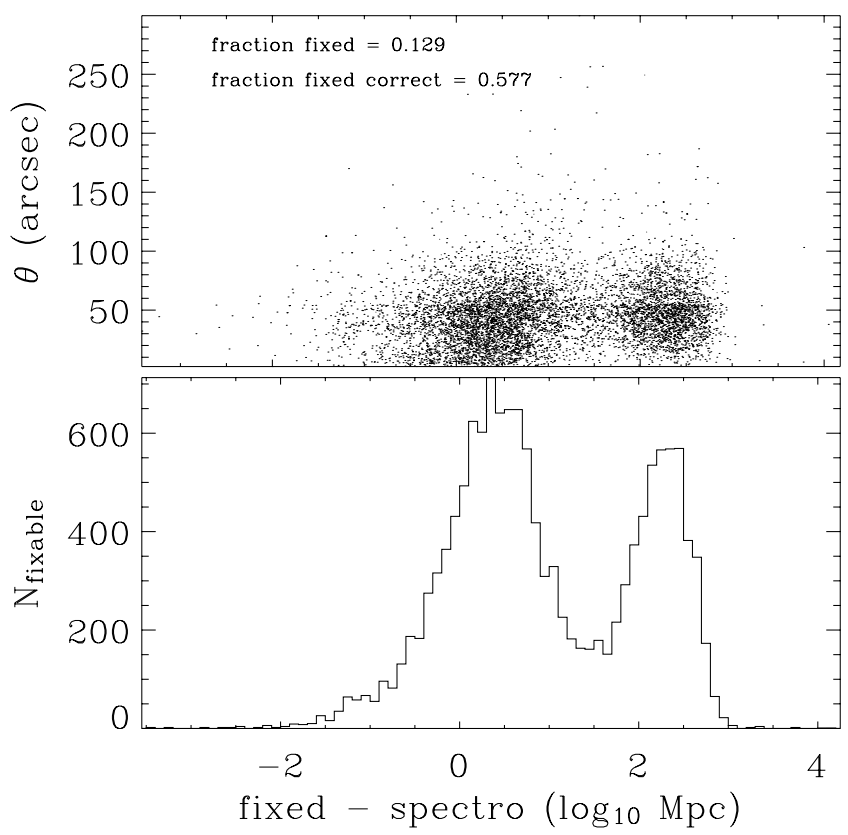

Fig. 8. - Top: Distribution of the redshift and angular differences of pairs of galaxies that could have been corrected using the procedure of $\S 4.1$ but in fact have an available redshift. Bottom: Histogram of the distribution of redshift differences.

These are in files of the form \$VAGC_REDUX/kcorrect/ kcorrect. \$collision. \$flux.z\$bandshift.fits, where \$collision refers to the type of collision correction (that is none, nearest, or photoz), $\$$ flux refers to the type of flux used for the SDSS data (on the basis of the prefix used in the calibObj files), and \$bandshift refers to the blueshift of the bandpasses we are correcting to. As an example, \$VAGC_REDUX/kcorrect/ kcorrect.none. petro.z0.10. fits contains corrections for galaxies that use no collision corrections, have Petrosian fluxes for the SDSS observations, and are shifted to the equivalent bandpass shapes at $z=0.10$.

\subsection{Sérsic Profile Fits}

In the file \$VAGC_REDUX/sersic/sersic_catalog.fits, we provide Sérsic fits to the azimuthally averaged radial profiles of each object (Sérsic 1968). Here we provide a description of the fitting procedure; a more detailed description can be found in the appendix of Blanton et al. (2005a).

For each galaxy, we fitted an axisymmetric Sérsic model of the form

$$
I(r)=A \exp \left[-\left(r / r_{0}\right)^{1 / n}\right]
$$

to the mean fluxes in the annuli outputted by the SDSS photometric pipeline photo in the quantities prof Mean and profErr (Stoughton et al. [2002] list the radii of these annuli). In equation (6), $n$ is referred to as the Sérsic index. The photo code outputs these quantities only out to the annulus that extends beyond twice the Petrosian radius, or to the first negative value of the mean flux, whichever is largest. In any case, we never consider data past the 12th annulus, whose outer radius is $68 . " 3$. For the median galaxy we have data and perform the fit out to 27.9 (the median half-light radius from the fits is $2^{\prime \prime}$ ). We minimize

$$
\chi^{2}=\sum_{i}\left\{\left[\operatorname{profMean}_{i}-\operatorname{sersicMean}_{i}\left(A, n, r_{0}\right)\right] / \operatorname{profErr}_{i}\right\}^{2}
$$




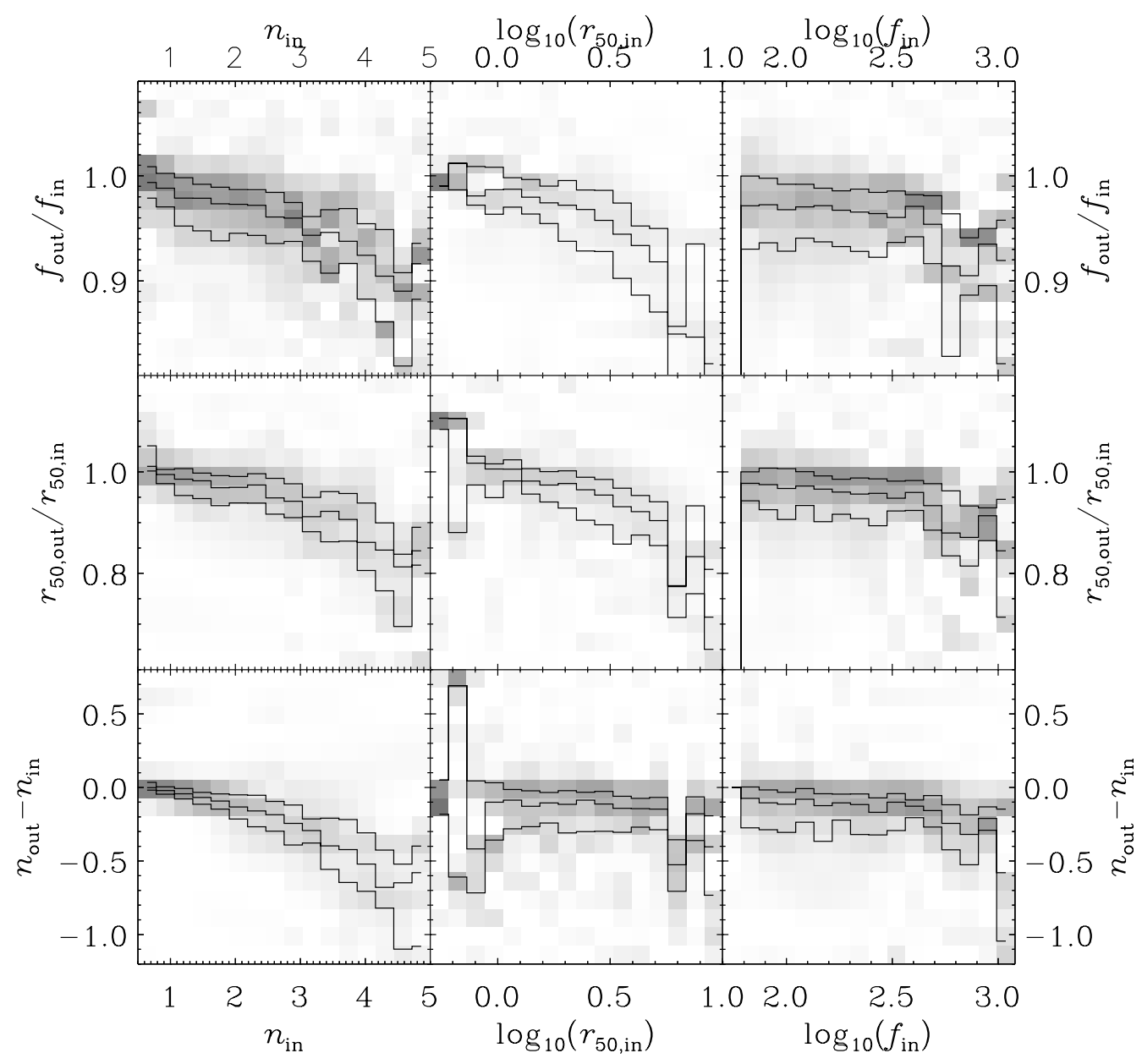

FIG. 9.-Residuals of the fitted Sérsic parameters as a function of the input Sérsic parameters for a set of 1200 simulated galaxies inserted into raw data and processed with the SDSS photometric pipeline and the Sérsic-fitting procedure. The fluxes and sizes are those associated with the Sérsic fit. The gray scale represents the conditional probability of the $y$-axis measurement given the $x$-axis input; the lines show the quartiles of that distribution.

where $\operatorname{sersicMean} i\left(A, n, r_{0}\right)$ is the mean flux in annulus $i$ for the Sérsic model convolved with a three-Gaussian seeing model for the given field.

We have evaluated the performance of this algorithm in the following way: Taking a sampling of the parameters of our fits from the Main galaxy sample, we have generated about 1200 axisymmetric fake galaxy images following equation (6) exactly, which we refer to as "fake stamps." In order to simulate the performance of photo, we have distributed the fake stamps among SDSS fields. For each band, we convert the fake stamps to SDSS raw data units, convolve with the estimated seeing from the photometric pipelines, and add Poisson noise using the estimates of the gain. We add the resulting image to the SDSS raw data at a random location on the frame, including the tiny effects of nonlinearity in the response and the less tiny flat-field variation as a function of column on the chip. We run photo on the resulting set of images to extract and measure objects and then run our Sérsic-fitting code. This procedure thus includes the effects of seeing, noise, and sky subtraction. We have tested that our results remain the same if we insert images using an alternative estimate of the seeing based simply on stacking nearby stars (still fitting using our three-Gaussian fit to the PSP seeing estimate).

Figure 9 displays the distribution of fit parameters in the $r$ band (converting $A$ and $r_{0}$ to total flux $f$ and half-light radius of the profile $r_{50}$ ) as a function of the input parameters. Each panel shows the conditional distribution of the quantity on the $y$-axis as a function of the quantity on the $x$-axis. The fluxes $f$ are expressed in nanomaggies, such that $f=100$ corresponds to $m=$ 17.5, near the flux limit of the Main galaxy sample. The lines show the quartiles of the distribution. At all Sérsic indices, sizes, and fluxes, the performance is good.

For larger sizes, sizes and fluxes are underestimated by about $10 \%$ and $15 \%$, respectively, while the Sérsic index is constant over a factor of 10 in size. For high Sérsic indices, the sizes and fluxes are slightly underestimated (again by about $10 \%$ and $15 \%$ ), while the Sérsic index itself is underestimated by (typically) -0.5 for a de Vaucouleurs galaxy, meaning that a true de Vaucouleurs $(n=4)$ galaxy yields $n \sim 3.5$ in our fits. This remaining bias is not much larger than the uncertainty itself and is comparable to the bias one expects (in the opposite direction) from neglecting nonaxisymmetry.

The bias is partly due to our approximate treatment of the seeing but mostly due to small errors in the local sky level (at the level of $1 \%$ or less of the sky surface brightness) determined by the photometric software. If one fits for the sky level, one can recover the Sérsic indices (and fluxes and sizes) of the fake galaxies far more accurately. However, because the Sérsic model is not a perfect model for galaxy profiles, for real data the fits apply unrealistically high changes to the sky level to attain slight decreases in $\chi^{2}$. The resulting sizes and fluxes of the largest and brightest galaxies are obviously wrong. Thus, we satisfy ourselves 
that the measurements we obtain with the fixed sky level yield approximately the right answer for galaxies that are actually Sérsic shaped, and for other galaxies merely supply a seeingcorrected estimate of size and concentration.

\subsection{Distances to Low-Redshift Galaxies}

At very low redshift we must take care in using the redshift as an estimate of the distance. First, we convert the heliocentric redshift provided by idlspec2d into the frame of the Local Group barycenter using the Local Group heliocentric velocity determination of Yahil et al. (1977). Then, we use a model of the local velocity field based on the IRAS 1.2 Jy redshift survey determined by Willick et al. (1997) (using $\beta=0.5$ ) in order to find the most likely distance of the given galaxy. Along the sight line $\hat{x}$ to each galaxy we maximize the likelihood density expressed by

$$
p(r)=r^{2} \exp \left\{\left[c z_{\mathrm{LG}}-v(r, \hat{\boldsymbol{x}})-r\right]^{2} / 2 \sigma_{v}^{2}\right\},
$$

where $v(r, \hat{\boldsymbol{x}})$ is the outward radial peculiar velocity at distance $r$ (expressed in $\mathrm{km} \mathrm{s}^{-1}$ ) in direction $\hat{\boldsymbol{x}}$. The fit of Willick et al. (1997) extends to $64 h^{-1} \mathrm{Mpc}$. Outside that radius we neglect peculiar velocities and assume the Hubble Law is exact. We set $\sigma_{v}=150 \mathrm{~km} \mathrm{~s}^{-1}$ independent of local density. We taper the peculiar velocities $v(r, \hat{x})$ to zero between 50 and $64 h^{-1} \mathrm{Mpc}$ in order to provide a smooth transition between these two regimes. The typical corrections are on the order of $200-300 \mathrm{~km} \mathrm{~s}^{-1}$.

We report errors in the distance based on the following calculation: we find the farthest distances above and below the bestfit distance at which the probability in equation (8) is equal to $\exp (-2)$ of its peak value (the $2 \sigma$ point in Gaussian statistics) and report one-quarter of the difference as the standard deviation in the distance. Near the edges of the volume for which we have an estimate of the velocity field ( $r=0$ and $6400 \mathrm{~km} \mathrm{~s}^{-1}$ ), we use half of the difference between the best-fit distance and the inner distance satisfying the above criterion. Outside that volume, we simply use the velocity dispersion $\sigma_{v}=150 \mathrm{~km} \mathrm{~s}^{-1}$.

In order to test our method, we have compared our version of distances to a set of galaxies in common with the Mark III catalog of Willick et al. (1997). Our results are consistent with the IRAS-predicted velocity field distances (dist_iras) in that catalog. On the other hand, there are significant disagreements (at the few megaparsec level) with the Tully-Fisher corrected distances (dist_tfc) of that catalog, in the sense that the Tully-Fisher distances tend to be higher. These differences reflect the inability of the IRAS density field to predict velocities perfectly in the directions probed by our sample.

The results of the procedure are in the file \$VAGC_REDUX/ velmod_distance/distance_sigv150.fits, which lists the coordinates and the heliocentric (ZACT), Local Group relative (ZLG), and peculiar velocity-corrected (ZDIST) redshifts for each object.

\subsection{Matching Spectra for Badly Deblended Targets}

When we matched the SDSS tiling catalog (sdss_tilingcatalog.fits) and the SDSS spectroscopic catalog (sdss_ spectro_catalog.fits) to the SDSS imaging catalog data, we used a match length of $2^{\prime \prime}$. However, for low surface brightness or complex galaxies, the behavior of the deblender has changed with the photometric software over time. Thus, the spectrum may reflect that of an object in the latest SDSS imaging catalog but not be near the nominal center of that object. We would like to make sure that we can recover the redshifts of objects in these cases.

In order to do so, we take all objects in the object_sdss_ imaging file that have no spectroscopic matches and compare them to all spectra in the SDSS spectroscopic catalog that are not already matched to object_sdss_imaging galaxies and that are within $2 r_{\mathrm{P}, 90}$ (twice the Petrosian 90\% light radius) of the center of the object. There are about 3000 spectra with such a candidate match. For each object we take a $3^{\prime \prime}$ radius aperture around the center of each nearby spectrum and measure the flux contributed by the object in question according to the deblender (or its parent, if the quality flag USE_PARENT is set), as well as the total flux in that exact same aperture. If the flux in that aperture from the object is at least half of the total flux in that aperture, then we consider the given spectrum to match the given object. We perform the same operation for the tiling catalog in order to match it to the object_sdss_imaging catalog.

We store the results in the files \$VAGC_REDUX/matchspec/ matchspec.fits and \$VAGC_REDUX/matchspec/matchtiled.fits. Each file contains an entry for the closest spectrum within $2 r_{\mathrm{P}, 90}$ of the center of each unmatched object. (The column OBJECT_POSITION indicates which object is under consideration, and the column ISP or ITI indicates which entry of the sdss_spectro_catalog or sdss_tiling_catalog.) The column SPMATCHED or TIMATCHED indicates whether the entry satisfies the criterion above. The catalog entries from sdss_ spectro_catalog or sdss_tiling_catalog are also included for convenience. The results of this operation are included when we build the large-scale structure sample ( $\S 4.8)$, the low-redshift catalog $(\S 5)$, the $K$-corrections ( $(4.2)$, and the collision corrections $(\S 4.1)$.

\subsection{Double Stars}

At very low redshifts, many of the photometrically defined "galaxies" are not galaxies at all but instead are double stars that the photometric software failed to deblend. Typically, these double stars are flagged as galaxies by the photometric pipelines, because they are resolved, bright $\left(m_{r}<16\right)$, and small $\theta_{50}<2^{\prime \prime}$.

In order to find many of these double stars, we have postprocessed the atlas images for all objects that the photometric software reports as resolved and that have $c z<1500 \mathrm{~km} \mathrm{~s}^{-1}$, as well as all galaxies without spectra for which $\mu_{50, r}<19$. We fitted a double point-spread function (PSF) model to the $r$-band image using an idlutils ${ }^{13}$ utility we wrote for this purpose called multi_psf_fit. We classify as double stars all objects that pass both the following criteria:

$$
\begin{gathered}
\frac{f_{\text {model }}}{f_{\text {PSF }}}<2, \\
\frac{\chi_{\text {double }}^{2}}{\chi_{\text {single }}^{2}}<0.2,
\end{gathered}
$$

where $f_{\text {model }}$ and $f_{\mathrm{PSF}}$ are the "model" and "PSF" fluxes reported by the photometric pipeline (Abazajian et al. 2004) and $\chi_{\text {double }}^{2}$ and $\chi_{\text {single }}^{2}$ are measurements from multi_psf_fit of the residuals between a model and the image using the double-star and single-star models. The first criterion is necessary to exclude cases in which the galaxy model fit is much more appropriate; the second criterion simply measures how much better

\footnotetext{
${ }^{13}$ See http://spectro.princeton.edu.
} 
TABLE 2

Eyeball Quality Flags

\begin{tabular}{|c|c|c|}
\hline Bit & Name & Description \\
\hline 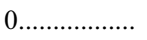 & DONE & We have checked this object \\
\hline 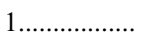 & OTHER & There is something wrong that does not fit any of the classes below \\
\hline $2 \ldots \ldots \ldots \ldots \ldots \ldots$ & UNCLASSIFIABLE & We cannot determine whether or not something is wrong \\
\hline 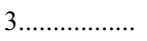 & NEED_BIGGER_IMAGE & We looked at too small an image to figure out what is going on \\
\hline 4..................... & BAD_DEBLEND & Ignore anything with this flag unless USE_ANYWAY or USE_PARENT is set \\
\hline 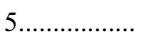 & FLECK & This object has only a tiny bit of the total flux of the full object \\
\hline 6..................... & DOUBLE_STAR & Double star \\
\hline 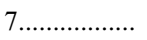 & HII & $\mathrm{H}$ II region plucked out of a larger galaxy \\
\hline 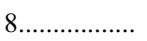 & USE_ANYWAY & There is a bad deblend, but we recommend you include the object in your sample anyway \\
\hline 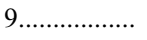 & EDGE & Something is wrong as a result of the object encountering an edge of a field \\
\hline 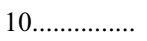 & SATELLITE & This object is actually a satellite flying overhead \\
\hline $11 \ldots \ldots \ldots \ldots \ldots . .$. & PLANE & This object is actually a plane flying overhead \\
\hline $12 \ldots \ldots \ldots \ldots . . . . .$. & BAD_Z & The listed redshift is wrong, and the spectro outputs think it is right \\
\hline 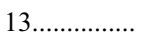 & INTERNAL_REFLECTION & Strongly affected by reflection inside the instrument \\
\hline 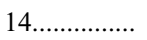 & BAD_SPEC_CLASS & The spectroscopic classification is wrong \\
\hline 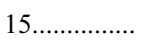 & USE_PARENT & We recommend the parent object of this bad deblend (see BAD_PARENT_CENTER below) \\
\hline 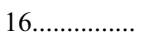 & IN_HUGE_OBJECT & Even the parent of this object is part of something even bigger \\
\hline 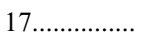 & STAR_ON_GALAXY & Star sitting in the foreground of a distant galaxy (resulting in a "galaxy" with a very small redshift) \\
\hline 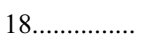 & QSO_ON_GALAXY & QSO in the background of a galaxy (resulting in a very high luminosity "galaxy") \\
\hline 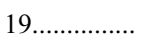 & NEGATIVE_QSO_FIT & Usually indicative of a redshift error \\
\hline 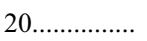 & BAD_SPECTRUM & The spectrum itself is bad (for example, contaminated by scattered light from a nearby bright star) \\
\hline 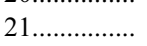 & POSSIBLE_LENS & This object is possibly a multiply imaged lens object \\
\hline 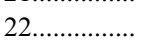 & IS_STAR & Although classified as a resolved object, this is definitely a star \\
\hline 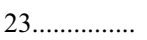 & DOUBLE_Z & The spectrum has two potential redshifts \\
\hline 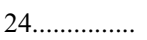 & PLANETARY_NEBULA & The spectrum is actually that of a planetary nebula in the foreground \\
\hline $25 \ldots \ldots \ldots \ldots \ldots \ldots$ & BAD_PARENT_CENTER & The parent is centered in an odd place, which will probably confuse structural measurements \\
\hline 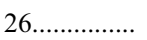 & GOOD_Z & The redshift reported by the SDSS spectroscopic pipeline is good, even though the ZWARNING value is nonzero \\
\hline
\end{tabular}

NoтE.-The names of the flags are in the idlutils file \$IDLUTILS_DIR/data/sdss/sdssMaskbits.par and can be accessed using the IDL functions sdss_flagname() and sdss_flagval() in idlutils (the bitmask name is Q_EYEBALL).

the double-star fit is. We have set the parameters conservatively in the sense that there are essentially no objects reported as double stars that are not in fact double stars. On the other hand, this conservatism means that there are some double stars not flagged as such by this procedure.

The results of this procedure exist in the file \$VAGC_REDUX/ doublestar/doublestar.fits. The important column in this file is ISDOUBLE, which has a " 1 " if the procedure above concludes that the object is a double star and " 0 " otherwise.

It is worthwhile noting that because $\mathrm{M}$ stars have such distinctive spectra, any resolved object flagged in object_sdss_ spectro as an M star is in fact a double star or an M star in the foreground of a distant galaxy. Therefore, when searching for very low redshift objects, one should exclude any object with a subclass of M. On the other hand, it is common for lowredshift galaxies to be classified as stars of other types.

\subsection{Eyeball Quality Checks}

For various purposes, we have performed a number of eyeball checks on the photometry and the spectra. We do not claim any completeness in terms of what set of objects we have checked, but it is usually productive to exclude objects we have flagged as errors in this list. The quality file is at \$VAGC_REDUX/ eyeball/quality.fits. For each object that we have quality checked, we have set values in a bitmask flag whose values and meanings are listed in Table 2. For objects with DONE set and no other flags, we have concluded that the object is dealt with by photo more or less correctly. Any other flag set indicates an error, and the object should be ignored, except for the cases in which USE_ANYWAY is set, which means we have concluded that keeping the object and its measurements is better than ex- cluding it, or in which USE_PARENT is set, which means we recommend using the measurement of the parent.

In cases of USE_PARENT, we have created a set of files for the measurement of the parents, which is at \$VAGC_REDUX/parents. This directory is more or less constructed to be parallel with the \$VAGC_REDUX directory. The list of objects whose parents we have processed is in the file \$VAGC_REDUX/parents/ object_sdss_imaging_parents.fits, which also has the photometric information for each object (as in the object_sdss_ imaging file). We calculate $K$-corrections and Sérsic fits for the parents and store them in the appropriate directories below this level, as fully documented on the Web site listed in Table 1.

In some cases, the parent is centered in an odd place, such as an $\mathrm{H}$ II region on the outskirts of the galaxy, rather than the center. These objects we flag as BAD_PARENT_CENTER. Although the redshifts and some photometric measurements will be fine, structural measurements (such as Sérsic fits) will be misleading.

\subsection{Large-Scale Structure Geometry}

In a separate directory, \$LSS_REDUX/drtwo14, we store the information we use to describe the large-scale structure geometry of the survey and with which we study large-scale structure. Recall that, like \$VAGC_REDUX, \$LSS_REDUX is an environmental variable describing the root location of the largescale structure data. The relevant value will be listed in the online documentation, as it will in principle change over time.

There are two basic files. First, there is one file fully describing the geometry, 1ss_combmask.drtwo14. fits, which is readable with the idlutils routine read_fits_polygons and which contains a row for every spherical polygon. This geometry is the area 
covered by the imaging, by the set of survey tiles, and not near Tycho stars (as described below).

1. SECTOR: Sector number (see description of tiling geometry in $\S 3.1 .2$ ).

2. FGOTMAIN: Fraction of Main sample targets that have good redshifts in this sector.

3. MMAX: Flux limit based on targeting limit and change in calibration since targeting ( $r$-band magnitudes).

4. DIFFRUN: Whether the "best" imaging in this field is the same observation as the "target" imaging.

5. ITILING_GEOMETRY: Position (zero-indexed) of the tiling polygon (see $\S 3.1 .2$ ) to which this polygon belongs.

6. ITARGET_GEOMETRY: Position (zero-indexed) of the target polygon to which this polygon belongs (in the file \$VAGC_ REDUX /sdss/sdss_target_geometry.fits).

7. ILSS: Index into the 1ss_geometry file described below.

8. RA: Estimated center of the field on which this was based (J2000.0 degrees).

9. DEC: Estimated center of the field on which this was based (J2000.0 degrees).

RA and DEC are not always inside the given polygon; however, the polygon is always fully contained within a 0.36 circle surrounding that center.

Note that MMAX varies with position for two reasons. First, the explicit targeting limits have changed with time over the course of the survey. Second, the calibration of the data has improved since the targeting, such that the flux limit in the recalibrated data varies across the sky. MMAX accounts for both effects.

There is an IDL utility in the vagc product called get_sdss icomb that takes right ascension and declination values and quickly checks which row of the 1ss_combmask file those coordinates are contained in. Because this piece of code is relatively simple and self-contained, we reproduce it in fully in the Appendix.

Second, there is a file describing the relationship that each object in object_sdss_imaging has with the geometry: 1ss_ index.drtwo14.fits. This file contains the following columns:

1. RA: Right ascension (J2000.0 degrees).

2. DEC: Declination (J2000.0 degrees).

3. Z: Redshift (from SDSS, 2dFGRS, $\mathrm{PSC} z$, or RC3, in that order of preference).

4. ZTYPE: Catalog (one-indexed) from the above list that the redshift comes from (zero if none).

5. SECTOR: Sector the object is in.

6. FGOTMAIN: Main sample completeness for each object (as described above).

7. MMAX: Nominal flux limit in the area of sky this object is in.

8. ILSS: Polygon in the lss_geometry file that this object is in ( -1 if none).

9. ICOMB: Polygon in the 1ss_combmask file that this object is in ( -1 if none).

10. VAGC_SELECT: How this object was selected (see $\S 3.1 .1$ ).

This file can be used to make some simple cuts to select galaxies with redshifts. The SDSS redshifts include those that have the ZWARNING flag set but that we have determined to be good and flagged as GOOD_Z (see $\S 4.7$ ), as well as those that we have matched using the extended matching criterion described in $\S 4.5$.

In addition, there are two files describing the geometry out of which we have built lss_combmask: lss_geometry. drtwo14.fits and lss_bsmask.drtwo14.fits. Each of these files contains sets of spherical polygons. The first file contains the geometry of the survey as a whole. The second file contains a mask cut out around bright stars (stars with $B<13$ in the Tycho catalog; Høg et al. 2000). The radius of the circle around each bright star is set according to the formula

$$
\theta=0.0802 B^{\prime 2}-1.86 B^{\prime}+11.625,
$$

where $\theta$ is in arcminutes; $B^{\prime}$ is the Tycho magnitude $B$ if $6<$ $B<11.5$, but $B^{\prime}=6$ if $B<6$, and $B^{\prime}=11.5$ if $B>11.5$. This radius is about that at which the mean density of galaxies near Tycho stars drops to half the background value (I. Strateva 2001, private communication). The mask is designed such that each polygon describing it is fully contained in a single polygon of the lss-geometry file (which one is stored in the ILSS column).

Finally, the directory \$LSS_REDUX/drtwo14/random/ contains 100 random catalogs with 1 million points each, distributed with constant surface density in the area included by lss_ combmask. In addition to right ascension and declination, this file contains ILSS, indicating which polygon of Iss_geometry the random point is in, and $\mathrm{EBV}$, the $E(B-V)$ value for this direction from Schlegel et al. (1998).

\section{A LOW-REDSHIFT CATALOG $(0.0033<z<0.05)$}

One of the areas of the SDSS that requires special care is the treatment of galaxies at low redshift. In order to study the property of galaxies at low redshifts and, correspondingly, at low luminosities, we have done some simple checks of the SDSS catalog in this regime, cleaned up the catalog where it was simple to do so, and put together a "low-redshift" catalog of galaxies with estimated comoving distances in the range $10 h^{-1} \mathrm{Mpc}<$ $d<150 h^{-1}$ Mpc.

For the purposes of this catalog, we checked the atlas images and spectra of a number of galaxies. We flagged bad deblends as errors and set other quality flags according to $\S 4.7$. In particular, in DR2 we have checked all objects in the catalog that we have selected as main sample-like objects (see $\S 3.1 .1$ ), that have a spectrum, and that satisfy one of the following criteria:

1. Having $M_{r}>-15$ and $z>0.003$, if a good redshift exists in the sample redshift range, the object is not classified as a double star according the algorithm in $\S 4.6$, and it is not classified as an M star. We deemed about $22 \%$ of these to be deblending errors in the latest reductions; for about $72 \%$ of these errors ( $16 \%$ of the total number) using the parent photometry is sufficient. Therefore, we recover about $94 \%$ of the objects in this category.

2. Having $0.003<z<0.01$, if a good redshift exists, the object is not classified as a double star according the algorithm in $\S 4.6$, and it is not a star. Again, about $19 \%$ are deblending errors, for $70 \%$ of which ( $14 \%$ of the total number) the parent photometry is sufficient, thus recovering about $96 \%$ of the galaxies in this category.

3. Having the spectroscopic pipeline warning flag ZWARNING set to something nonzero, indicating a problem. For many of these (about 25\%) the software in fact finds a correct redshift, and we flag these as GOOD_Z (see Table 2). This yields 97 more galaxies in our sample.

4. Being in a pair with a separation less than $50 h^{-1} \mathrm{kpc}$ and an angular separation less than 0.1 . About $12 \%$ of these are incorrectly deblended in this sample, about $37 \%$ of which $(4 \%$ of the full sample) could be replaced with the parent photometry, yielding a recovery rate of $92 \%$ of the galaxies in this category. 
Thus, after performing these checks, we are correctly treating the photometry for more than $90 \%$ of the galaxies for which we have spectroscopy.

We select galaxies from the NYU-VAGC for the low-redshift sample according to the following criteria:

1. The galaxy is selected by the Main sample-like criterion defined in $\S 3.1 .1$ (that is, the bitmask VAGC_SELECT has bit 2 set).

2. The quality bit has no flags set except for DONE and/or GOOD_Z or BAD_PARENT_CENTER, or it has USE_ANYWAY or USE_PARENT set.

3. The spectroscopic subclass does not indicate an $\mathrm{M}$ star.

4. We have not classified the object as a double star (see $\S 4.6)$.

5. There is a good SDSS redshift determination (meaning the spectroscopic flag ZWARNING was zero or the quality flag GOOD_Z was nonzero).

6. The distance estimate from $\S 4.4$ yields a distance redshift within the range $0.0033<z<0.05$ (see below).

7. The object is within the large-scale structure geometry and outside the bright star mask (see $\S 4.8$ ).

8. The Galactic extinction-corrected, $r$-band Petrosian magnitude is less than the flux limit in the given direction (see $\S 4.8$ ).

The redshifts we use include those matched to the imaging objects using the criterion described in $\S 4.5$. Note that we do not require that the object be spectroscopically classified as a galaxy; a certain number of low-redshift galaxies are classified as stars, especially in cases in which the spectrum has a low signal-to-noise ratio. The low-redshift cut corresponds to $10 \mathrm{~h}^{-1}$ Mpc. We do not consider anything below this redshift because the sample becomes highly incomplete (as a result of shredding by the photometric pipeline of large resolved galaxies) and the distance estimates for such objects are highly affected by peculiar velocities.

For galaxies with USE_PARENT set, we replace the SDSS child's photometry with that of the parent, using the results described in $\S 4.7$. In the DR2 area, 28,089 galaxies pass the above criteria. Weighted by the completeness, the effective area of the sample is $2220.9 \mathrm{deg}^{2}$.

To compute the global number densities of galaxies as a function of their properties, it is necessary to compute the numberdensity contribution $1 / V_{\max }$ for each galaxy, where $V_{\max }$ is the volume covered by the survey in which this galaxy could have been observed, accounting for the flux, redshift limits, and completeness as a function of angle (Schmidt 1968). We calculate this volume as follows:

$$
V_{\max }=\int d \Omega f(\theta, \phi) \int_{z_{\min }}^{z_{\max }(\theta, \phi)} d z \frac{d V}{d z}
$$

where $f(\theta, \phi)$ is the spectroscopic completeness as a function of angle, averaging about $90 \%$ across the survey. We determine this on a sector-by-sector basis (it is the FGOTMAIN value described in $\S 4.8)$. In addition, $z_{\max }(\theta, \phi)$ is defined for this sample by

$$
\begin{gathered}
z_{\max }(\theta, \phi)=\min \left[z_{m, \max }(\theta, \phi), 0.05\right], \\
z_{\min }=0.0033 .
\end{gathered}
$$

The flux limit $m_{r, \max }(\theta, \phi)$ (usually $17.77 \mathrm{mag}$ ) implicitly sets $z_{m,\{\min , \max \}}(\theta, \phi)$ by

$$
\begin{aligned}
m_{r, \text { max }}(\theta, \phi)= & M_{r}+\mathrm{DM}\left(z_{m, \text { max }}(\theta, \phi)\right) \\
& +K_{r}\left(z_{m, \text { max }}(\theta, \phi)\right) .
\end{aligned}
$$

Note that over this redshift range we can ignore the contribution of the surface brightness limits to $V_{\max }$, since cosmological surface brightness dimming is such a small effect.

There is a complication at low redshift: our estimate of the luminosity has a large uncertainty as a result of galaxy peculiar velocities, and $V_{\max }$ as calculated above is a nonlinear function of luminosity, such that an uncertainty in luminosity will yield an underestimate of $V_{\max }$. For a fair estimate of $V_{\max }$, we use

$$
V_{\max }=\int d L p(L) V_{\max }(L)
$$

where $p(L) d L=p(r) d r$ (from eq. [8] above). This estimate is an average of $V_{\max }$ for all possible luminosities based on the probability of the galaxy having that luminosity. In practice, there is only a small difference between the results one finds using the regular $V_{\max }$ estimator and this one.

The catalog is available in the file \$VAGC_REDUX/lowz/ lowz_catalog.drtwo14.fits. Its columns are described in Table 3.

Atlas images (that is, images with neighboring objects removed; Stoughton et al. 2002) of all the objects in the catalog are available in the directories \$VAGC_REDUX/lowz/images/ 00h, \$VAGC_REDUX/lowz/images/01h, . . , \$VAGC_REDUX/ lowz/images $/ 23 \mathrm{~h}$, where each directory contains galaxies in a particular hour of right ascension (J2000.0). The names of each atlas image are based on the IAU name of each object: e.g., lowz-atlas-J044112.00+003202.3.fits. As described on the Web site, each file contains 10 HDUs. HDUs $0,2,4,6$, and 8 contain the ugriz images of each galaxy. HDUs $1,3,5,7$, and 9 contain the inverse variance ugriz images of each galaxy. In addition, there is a file of the form psf-J044112.00+ 003202.3. fits, which contains five HDUs, the estimated PSFs at the center of the object in ugriz.

We have already used this low-redshift catalog for an investigation of the population of low-luminosity galaxies in the SDSS survey (Blanton et al. 2005b). We expect the catalog and images to be useful in a number of other ways. For example, it provides a nice low-redshift sample for comparison to highredshift galaxy samples.

\section{SOFTWARE TOOLS}

Generally speaking, we have used a combination of $\mathrm{C}$ and IDL in constructing this catalog. Astronomers will likely find many of the utilities we have used to construct the data set useful for analyzing it as well. The locations for source code and online documentation for some of these tools are listed in Table 1.

Here are some short descriptions of the tools themselves:

1. idlutils: A general astronomically useful set of IDL utilities maintained by D. Schlegel and D. Finkbeiner, incorporating the Goddard IDL library maintained by W. Landsman and contributed to by many others too numerous to mention. In particular, this library contains readers for the FITS files, spherical polygon files (see $\S 2$ below), and FTCL parameter files (a special ASCII format) that our catalog contains.

a) mrdfits is a general FITS reader that can read FITS images and tables (.fits files).

b) read_mangle_polygons reads spherical polygon files produced by mangle (.ply files), and read_fits_polygons reads spherical polygon files in FITS format (idlutils has code that makes it easy to work with the resulting structures). See the 
TABLE 3

Parameters in the Low-Redshift Catalog

\begin{tabular}{|c|c|}
\hline Column & Description \\
\hline OBJECT_POSITION ........... & Index of object in full NYU-VAGC \\
\hline 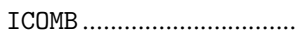 & Index indicating location in SDSS geometry (see $\S 4.8$ for details) \\
\hline SECTOR ............................. & Sector number in SDSS survey geometry (used to calculate completeness; see $\S 4.8$ for details) \\
\hline VAGC_SELECT..................... & Bitmask indicating how this object was selected (see $\S 3.1 .1$ for details) \\
\hline FGOTMAIN ........................... & Completeness of SDSS Main sample in this sector [that is, $f(\theta, \phi)$ ] \\
\hline 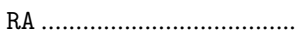 & Right ascension, J2000.0 (deg) \\
\hline 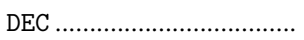 & Declination, J2000.0 (deg) \\
\hline RUN & SDSS drift scan number \\
\hline RERUN ............................... & SDSS processing rerun \\
\hline CAMCOL ............................... & SDSS camera column \\
\hline FIELD …............................ & SDSS field \\
\hline 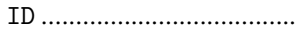 & SDSS object ID within the field \\
\hline OBJC_ROWC........................... & CCD $y$-position in the field (centers of pixels are half-integers) \\
\hline OBJC_COLC......................... & CCD $x$-position in the field (centers of pixels are half-integers) \\
\hline PLATE ................................ & SDSS spectroscopic plate \\
\hline FIBERID .............................. & SDSS spectroscopic fiber number \\
\hline 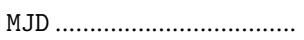 & Date of SDSS spectroscopic observation \\
\hline QUALITY ........................... & Eyeball quality flag (see $\S 2$ for full description) \\
\hline ABSMAG [8] ........................ & $\begin{array}{l}\text { Absolute magnitude (AB) in the ugrizJHK } K_{s} \text { bandpasses (first five from SDSS Petrosian magnitude, } \\
\text { last three from 2MASS XSC converted to the AB system as described in the text), } K \text {-corrected and } \\
\text { Galactic extinction-corrected (Schlegel et al. 1998) }\end{array}$ \\
\hline ABSMAG_IVAR [8] ............ & Inverse variance of uncertainty in absolute magnitude (including the effects of errors in the distance) \\
\hline 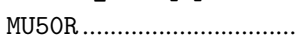 & Petrosian half-light surface brightness (mag $\operatorname{arcsec}^{-2}$ ) \\
\hline KCORRECT [8] ..................... & Estimated $K$-corrections in ugrizJHK $(\mathrm{mag})$ \\
\hline PETROFLUX [5] .................... & SDSS Petrosian flux in ugriz (nanomaggies; see eq. [4]) \\
\hline PETROTHETA [5] .............. & SDSS Petrosian radius in ugriz $(\operatorname{arcsec})$ \\
\hline PETROTH50 [5] .................. & SDSS Petrosian $50 \%$ light radius in ugriz (arcsec) \\
\hline PETROTH90 [5] .................. & SDSS Petrosian $90 \%$ light radius in ugriz (arcsec) \\
\hline EXTINCTION [5] .............. & Galactic extinction from Schlegel et al. (1998) in ugriz (mag) \\
\hline 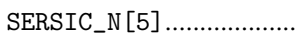 & Sérsic index estimated from radial profile in ugriz \\
\hline SERSIC_TH50 [5] ............ & $50 \%$ light radius from Sérsic fit in ugriz (arcsec) \\
\hline SERSIC_FLUX [5] ............. & Total flux from Sérsic fit in ugriz (nanomaggies; see eq. [4]) \\
\hline 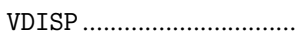 & Estimated velocity dispersion from spectrum \\
\hline 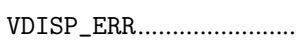 & Estimated uncertainty in velocity dispersion from spectrum \\
\hline CLASS ................................ & $\begin{array}{l}\text { Spectroscopic classification, as outputted by the SDSS spectroscopic pipeline (note that occasionally this is } \\
\text { incorrect; in particular, a number of galaxies in our sample are classified as stars spectroscopically because } \\
\text { the signal-to-noise ratio of the spectrum does not allow reliable discrimination between the two) }\end{array}$ \\
\hline SUBCLASS ......................... & Spectroscopic subclassification (e.g., stellar type), as outputted by the SDSS spectroscopic pipeline \\
\hline 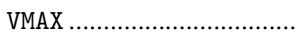 & Maximum volume in the sample over which we could have observed this object \\
\hline NEDNAME ............................... & Name of NED match \\
\hline 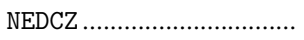 & Redshift from NED match \\
\hline ZLG ................................... & Local Group relative redshift from SDSS \\
\hline ZDIST .............................. & Peculiar velocity-corrected Local Group relative redshift from SDSS \\
\hline ZDIST_ERR ............................ & Uncertainty in ZDIST \\
\hline
\end{tabular}

Note.-These are the parameters included in the catalog described in $\S 5$.

mangle Web site listed in Table 1 or see $\S 2$ for details on spherical polygon files.

c) yanny_readone is an FTCL parameter file reader (.par files, a special SDSS ASCII format; see the DR2 Web site listed in Table 1 for details).

2. photoop: An SDSS-specific set of utilities written in Perl, IDL, and C by D. Schlegel, D. Finkbeiner, and N. Padmanabhan. These tools are primarily for performing the photometric reductions and calibrations but also contain image analysis utilities that may be useful to users.

3. vagc: The code responsible for producing this catalog. It contains some useful utilities for reading in the data from the catalog.

4. kcorrect: A set of utilities for calculating $K$-corrections and photometric redshifts, tuned to work for SDSS and 2MASS data (Blanton et al. 2003b). Our catalog contains $K$-corrections already calculated, but the user may find this useful.

5. mangle: A general set of tools for handling window functions on the sphere, developed by Hamilton \& Tegmark (2004). It is described more fully in $\S 2$. These routines can be useful to the user for creating random catalogs from the geometric descriptions given here, and also for checking whether certain directions are inside or outside the surveys. This code is also distributed as part of idlutils.

We should note that none of these tools are necessary for using the data, only recommended as generally useful packages.

\section{SUMMARY}

Here we have presented a catalog of galaxies combining information from SDSS, 2MASS, FIRST, $\mathrm{PSC} z, \mathrm{RC} 3$, and 


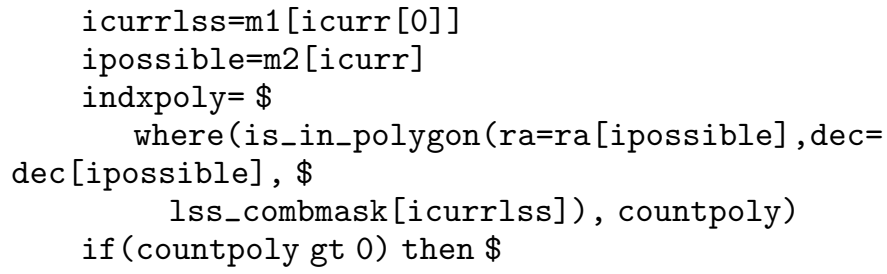

Abazajian, K., et al. 2003, AJ, 126, 2081 2004, AJ, 128, 502

Baldry, I. K., Glazebrook, K., Brinkmann, J., Ivezić, Ž., Lupton, R. H., Nichol, R. C., \& Szalay, A. S. 2004, ApJ, 600, 681

Becker, R. H., White, R. L., \& Helfand, D. J. 1995, ApJ, 450, 559

Blanton, M. R., Eisenstein, D. J., Hogg, D. W., Schlegel, D. J., \& Brinkmann, J. 2005a, ApJ, in press (astro-ph/0310453)

Blanton, M. R., Lin, H., Lupton, R. H., Maley, F. M., Young, N., Zehavi, I., \& Loveday, J. 2003a, AJ, 125, 2276

Blanton, M. R., et al. 2003b, AJ, 125, 2348 2003c, ApJ, 592, 819 2005b, ApJ, submitted

Colless, M., et al. 2001, MNRAS, 328, 1039

Cutri, R. M., et al. 2000, The 2MASS Explanatory Supplement (Pasadena: IPAC), http://www.ipac.caltech.edu/2mass/releases/allsky/doc/explsup.html de Vaucouleurs, G., de Vaucouleurs, A., Corwin, H. G., Buta, R. J., Paturel, G., \& Fouque, P. 1991, Third Reference Catalogue of Bright Galaxies (Berlin: Springer)

Eisenstein, D. J., et al. 2001, AJ, 122, 2267

Finlator, K., et al. 2000, AJ, 120, 2615

Fukugita, M., Ichikawa, T., Gunn, J. E., Doi, M., Shimasaku, K., \& Schneider, D. P. 1996, AJ, 111, 1748

Gunn, J. E., et al. 1998, AJ, 116, 3040

Hamilton, A. J. S., \& Tegmark, M. 2004, MNRAS, 349, 115

Høg, E., et al. 2000, A\&A, 355, L27

Hogg, D. W., Finkbeiner, D. P., Schlegel, D. J., \& Gunn, J. E. 2001, AJ, 122, 2129

Hogg, D. W., et al. 2003, ApJ, 585, L5

Hoyle, F., Rojas, R. R., Vogeley, M. S., \& Brinkmann, J. 2005, ApJ, 620, 618

Jarrett, T. H., Chester, T., Cutri, R., Schneider, S. E., \& Huchra, J. P. 2003, AJ, 125,525

\author{
icomb [ipossible [indxpoly] ]=icurrlss \\ istart=iend $+1 \mathrm{~L}$ \\ endf or \\ return, icomb \\ end
}

\section{REFERENCES}

Kurucz, R. L. 1991, in Precision Photometry: Astrophysics of the Galaxy, ed. A. G. D. Philip \& A. R. Upgren (Schenectady: Davis), 27

Lupton, R. H., Gunn, J. E., Ivezić, Z., Knapp, G. R., Kent, S., \& Yasuda, N. 2001, in ASP Conf. Ser. 238, Astronomical Data Analysis Software and Systems X, ed. F. R. Harnden, Jr., F. A. Primini, \& H. E. Payne (San Francisco: ASP), 269

Maddox, S. J., Efstathiou, G., \& Sutherland, W. J. 1990, MNRAS, 246, 433

Oke, J. B., \& Sandage, A. 1968, ApJ, 154, 21

Pier, J. R., Munn, J. A., Hindsley, R. B., Hennessy, G. S., Kent, S. M., Lupton, R. H., \& Ivezić, Ž. 2003, AJ, 125, 1559

Pope, A. C., et al. 2004, ApJ, 607, 655

Richards, G., et al. 2002, AJ, 123, 2945

Rudnick, G., et al. 2003, ApJ, 599, 847

Saunders, W., et al. 2000, MNRAS, 317, 55

Schlegel, D. J., Finkbeiner, D. P., \& Davis, M. 1998, ApJ, 500, 525

Schmidt, M. 1968, ApJ, 151, 393

Sérsic, J. L. 1968, Atlas de Galaxias Australes (Cordoba: Obs. Astronómico) Shen, S., Mo, H. J., White, S. D. M., Blanton, M. R., Kauffmann, G., Voges, W., Brinkmann, J., \& Csabai, I. 2003, MNRAS, 343, 978

Skrutskie, M. F., et al. 1997, in The Impact of Large Scale Near-IR Sky Surveys, ed. F. Garzón (Dordrecht: Kluwer), 25

Smith, J. A., et al. 2002, AJ, 123, 2121

Stoughton, C., et al. 2002, AJ, 123, 485

Strauss, M. A., et al. 2002, AJ, 124, 1810

Tegmark, M., Hamilton, A. J. S., \& Xu, Y. 2002, MNRAS, 335, 887

Tegmark, M., et al. 2004, ApJ, 606, 702

Trujillo, I., et al. 2004, ApJ, 604, 521

Willick, J. A., Strauss, M. A., Dekel, A., \& Kolatt, T. 1997, ApJ, 486, 629

Yahil, A., Tammann, G. A., \& Sandage, A. 1977, ApJ, 217, 903

York, D., et al. 2000, AJ, 120, 1579

Zehavi, I., et al. 2002, ApJ, 571, 172 